\title{
EFFECTS OF ADDED ELECTROLYTES ON THE STRUCTURE OF CHARGED POLYMERIC MICELLES
}

\author{
S. Mochizuki, S. Komura, and T. Kato $\square \quad$ Department of Chemistry, \\ Tokyo Metropolitan University, Tokyo, Japan
}

\begin{abstract}
$\square \quad$ We investigate the effects of added electrolytes on the structure of spherical aggregates made of neutral/charged diblock copolymers with weakly acidic groups on the charged block. Using a simple model based on the theory of annealed polyelectrolyte brushes, we study the added-electrolyte effects that differ according to the magnitude of the ionic strength and the acid concentration. They are presented by the regime diagrams as a function of the ionic strength and the acid concentration. Depending on the neutral/charged relative block length, six different types of regime diagrams are obtained. We also discuss the poor solvent case where the backbone of the charged block is highly hydrophobic. As a result of the competition between the osmotic repulsion and the hydrophobic attraction, the addition of salt induces a reentrant change of the structural parameters.
\end{abstract}

Keywords Macromolecular and polymer solutions, Polymer melts, Swelling, Supramolecular and biochemical assembly, Polyelectrolytes

\section{INTRODUCTION}

Charged diblock copolymers composed of a neutral block and a charged block self-assemble to form micelles in solvents such as water or water-methanol mixtures that are selective for charged blocks. In a dilute aqueous solution without added salt, micelles are often spherical, consisting of the core made up of neutral blocks and the corona composed of extended ionic blocks. Their structural properties have been investigated both experimentally and theoretically in order to understand the properties of polyelectrolyte brushes and the mechanism of colloidal stabilization by polyelectrolytes $(1,2)$.

In the previous theoretical approaches, charged blocks in the corona region are described as polyelectrolyte brushes (i.e., monolayers composed of polyelectrolytes that are grafted at one end onto an impermeable surface) (3-11). Polyelectrolyte brushes are classified into two types: quenched

Received April 5, 2006; Accepted May 8, 2006.

Address correspondence to S. Komura, Department of Chemistry, Faculty of Science, Tokyo Metropolitan University, Tokyo 192-0397, Japan. E-mail: komura@comp.metro-u.ac.jp 
brushes and annealed brushes (12). For quenched brushes, such as polystyrene sulfonate brushes, the number and position of charges on the grafted chain are fixed. For annealed brushes, on the other hand, they are controlled by the external conditions, such as $\mathrm{pH}$. These brushes are made of polyacids (or polybases) with weakly dissociating groups such as carboxyl groups ($\mathrm{COO}^{-} \mathrm{H}^{+}$). The fact that the ionization degree depends on the external conditions leads to nontrivial behaviors of annealed brushes. For example, the thickness of annealed brushes increases with the ionic strength at very low ionic strength rather than shrinking due to the screening effect (13-16).

For charged micellar solutions, the addition of electrolytes such as salt or acid changes the size of micelles (17-22) or induces morphological changes (22-26). Such phenomena occur due to the weakening of the electrostatic interactions between the corona chains. In dilute aqueous solutions of polystyrene- $b$-poly(acrylic acid) (PS-PAA) copolymers, for example, the addition of micromolar $\mathrm{HCl}$ or millimolar $\mathrm{NaCl}$ can cause the morphology change of PS-PAA micelles (22). On increasing the electrolyte concentration, the morphology changes from spheres to rods, vesicles, and then to large compound vesicles. The effects of added electrolytes depend on whether the charged blocks are quenched or annealed. For the quenched case, the effects of added salt are similar to those of added acid, because both of them localize near the charges on the corona chains (screening effect). For the annealed case, the addition of salt causes the promotion of dissociation at very low ionic strength, or the shielding of the ionized corona chains at relatively high ionic strength (13-16). Added acid, on the other hand, reduces the ionization degree of the corona chains by protonating the ionic sites.

Previously, the effects of added salt or acid on charged micellar solutions were investigated theoretically $(5,8-11)$. It was predicted that, for quenched copolymers, the aggregation number is constant at very low salt concentration, but it increases as $C_{\mathrm{S}}^{6 / 11}$ above a threshold salt concentration (9). This behavior is in qualitative agreement with the result of the scattering experiment on the aqueous solutions of polyethylethylene- $b$-poly(styrenesulfonic acid) (20). For annealed copolymers, the coupling between the ionization and the aggregation of copolymers was found theoretically. At very low ionic strength, for instance, the aggregation number decreases with increasing the salt concentration due to the promotion of dissociation $(10,11)$. Such a feature is not exhibited by quenched copolymers.

The purpose of the present paper is to investigate the effects of added electrolytes on the structure of charged micelles. We consider aggregates of diblock copolymers composed of a neutral block and a charged block with weakly acidic groups (i.e., annealed block). The micelles are assumed to be spherical, as observed in many experiments. The dependences of their structural parameters on the salt and acid concentrations are investigated using a simple model based on the theory of annealed polyelectrolyte brushes. Generally speaking, the interactions between the charged 
monomers in a polar solvent include not only the electrostatic repulsion but also the hydrophobic interaction, because the backbone of the charged block is made of hydrophobic hydrocarbons. Nevertheless, when the hydrophobicity of the blocks is not too large, the hydrophobic attraction can be neglected with respect to the electrostatic contribution. For such a case $(\theta-$ solvent case), we perform a scaling analysis to investigate the effects of added electrolytes on micellar structure. We find that the effects of electrolytes depend not only on the magnitude of the ionic strength and the acid concentration, but also on the neutral/charged relative block length. On the other hand, when the charged block is highly hydrophobic (e.g., poly(methacrylic acid) block with $\alpha$-methyl groups), we need to deal with the poor solvent case. As a result of the competition between the electrostatic repulsion and the hydrophobic attraction, we predict a reentrant change of the micellar structure caused by the addition of salt.

This paper is organized as follows. In the next section, we introduce a model for a spherical micelle. In the third section, critical micelle concentration is derived by minimizing the free energy of a micellar solution. In the fourth and fifth sections, we perform a scaling analysis for the structural parameters of crew-cut micelles and star-like micelles, respectively. In the sixth section, various regimes characterized by different scaling expressions are organized into six types of regime diagrams. In the seventh section, we numerically calculate the dependences of the structural parameters on the electrolyte concentration for the poor solvent case. Conclusions and discussion are provided in the final section.

\section{FREE ENERGY OF A SPHERICAL MICELLE}

Consider a charged diblock copolymer composed of a neutral $A$ block and a charged $B$ block in a selective solvent that is incompatible with the $A$ block. The polymerization degrees of the $A$ and $B$ blocks are denoted by $N_{A}$ and $N_{B}$, respectively. We assume that each repeating unit of the $B$ block possesses a weakly acidic group such as a carboxyl group $\left(-\mathrm{COO}^{-} \mathrm{H}^{+}\right)$. The dissociation degree of the acidic groups $\alpha$ depends on the local concentration of protons $c_{\mathrm{H}}$ :

$$
\frac{\alpha}{1-\alpha}=\frac{K_{a}}{c_{\mathrm{H}}},
$$

where $K_{a}$ is the dissociation constant.

When the concentration of the copolymers exceeds a threshold value called the critical micelle concentration (CMC), molecules self-assemble to form micelles. CMC is typically so small that the intermicellar interaction can be neglected. Micelles are considered to be spherical as observed in many experiments $(2,18-23)$. As shown in Fig. 1, they consist of a core 


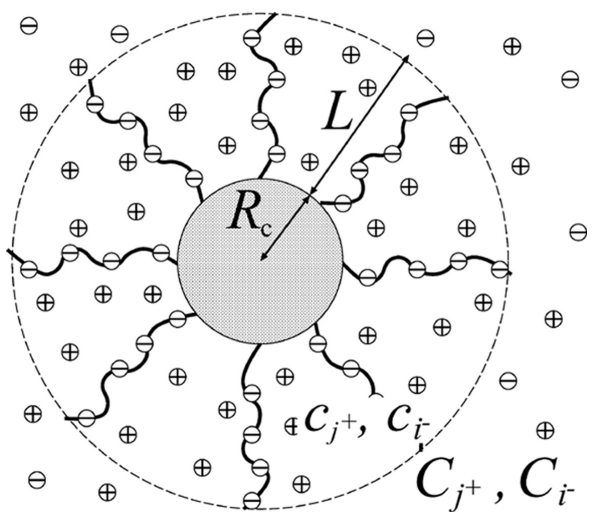

FIGURE 1 A spherical micelle. It consists of a core region (radius $R_{\mathrm{c}}$ ) made up of neutral blocks and a surrounding corona region (thickness $L$ ) where charged blocks are stretched. The concentration of mobile ions of species $i$ inside the corona region and in the solution are denoted by $c_{i}$ and $C_{i}$, respectively.

region made up of the neutral $A$ blocks and a surrounding corona region in which the charged $B$ blocks are stretched. We assume that the interface between these two regions is sharp and that the core region is incompressible. The latter condition is expressed as

$$
\frac{4}{3} \pi R_{\mathrm{c}}^{3}=p N_{A} a^{3}
$$

where $R_{\mathrm{c}}$ is the core radius, $p$ the aggregation number, and $a$ the size of the repeating unit of the copolymer. In the corona region, the concentration of the $B$ monomers is given by

$$
c_{\mathrm{p}}=\frac{N_{B}}{\Omega_{B}}=\frac{N_{B}}{a^{3} N_{A}}\left[\left(1+\frac{L}{R_{\mathrm{c}}}\right)^{3}-1\right]^{-1},
$$

where $\Omega_{B}$ is the volume of the corona per chain, and $L$ is the corona thickness. Hence, $L$ is expressed as a function of $c_{\mathrm{p}}$ by

$$
L=R_{\mathrm{c}}\left[\left(1+\frac{N_{B}}{a^{3} N_{A} c_{\mathrm{p}}}\right)^{1 / 3}-1\right] .
$$

The total free energy of a spherical micelle per chain is written as a sum of three contributions $(5,6,8-11,27,28)$ :

$$
f=f_{\text {core }}+f_{\text {interface }}+f_{\text {corona }} .
$$

The core energy $f_{\text {core }}$ represents the elastic energy per $A$ block:

$$
f_{\text {core }}=\frac{3 R_{\mathrm{c}}^{2}}{2 N_{A} a^{2}} .
$$


Here and in the following, all energies are expressed in units of the thermal energy $k_{\mathrm{B}} T$. In the above, we have assumed that each $A$ block is uniformly stretched so that its end-to-end distance is equal to the core radius $R_{\mathrm{c}}$. The second term $f_{\text {interface }}$ represents the interfacial energy between the core and the corona regions:

$$
f_{\text {interface }}=\gamma \frac{4 \pi R_{\mathrm{c}}^{2}}{p}=\frac{3 \gamma N_{A} a^{3}}{R_{\mathrm{c}}},
$$

where $k_{B} T \gamma$ is the interfacial tension. The value of $\gamma a^{2}$ is typically of order unity. We neglect the interfacial energy between the corona and the outer solution, because it is much smaller than the core-corona surface energy. The form of the corona energy $f_{\text {corona }}$ will be discussed in the following.

We assume that the corona chains are grafted on the core-corona interface to form an annealed polyelectrolyte brush (10-14). We then adapt the model of annealed polyelectrolyte brushes given in Ref. (13). All the corona chains are assumed to be uniformly stretched up to the corona thickness $L$. The corona region is surrounded by the solution of acid and salt. We require that the electroneutrality condition is satisfied both in the corona region and in the outer solution. The concentration of each component is taken to be uniform.

Let $c_{i}$ and $C_{i}$ be the concentration of mobile ions of species $i$ in the corona region and the outer solution, respectively. For simplicity, we assume that the weakly acidic group, acid, and salt are all monovalent. The electroneutrality conditions in the corona region and the outer solution are written as

$$
\begin{aligned}
\alpha c_{\mathrm{p}}+\sum_{i} c_{i^{-}} & =\sum_{j} c_{j^{+}}, \\
\sum_{i} C_{i^{-}} & =\sum_{j} C_{j^{+}},
\end{aligned}
$$

respectively, where $\alpha$ is the dissociation degree defined in Eq. (1). The mobile ions are distributed according to the Boltzmann's law:

$$
\begin{aligned}
c_{i^{-}} & =C_{i^{-}} \exp \left(\frac{e \Phi}{k_{\mathrm{B}} T}\right), \\
c_{j^{+}} & =C_{j^{+}} \exp \left(-\frac{e \Phi}{k_{\mathrm{B}} T}\right),
\end{aligned}
$$

where $e$ is the elementary charge, and $\Phi$ is the average electrostatic potential in the corona region with respect to the outer solution. Notice that $\Phi<0$ owing to the negative charges on the corona chains. Hence, the proton concentration in the corona region is higher than that in the 
outer solution (i.e., $c_{\mathrm{H}}>C_{\mathrm{H}}$ ) [see Eq. (11)]. We then obtain from Eq. (1)

$$
\alpha<\frac{K_{a}}{C_{\mathrm{H}}} .
$$

Thus, the dissociation degree of the corona chains is smaller than that of the isolated charged blocks in the outer solution. Eqs. (10) and (11) give rise to the so-called Donnan rule $(29,30)$ :

$$
\frac{C_{j^{+}}}{c_{j^{+}}}=\frac{c_{i^{-}}}{C_{i^{-}}} .
$$

Using Eqs. (1), (8), (9), and (13), we obtain a relation that determines $\alpha$ :

$$
\frac{K_{a}}{C_{\mathrm{H}}} \frac{1-\alpha}{\alpha}-\frac{C_{\mathrm{H}}}{K_{a}} \frac{\alpha}{1-\alpha}=\frac{\alpha c_{\mathrm{p}}}{C_{\mathrm{i}}} .
$$

Here, $C_{\mathrm{i}}=\sum_{j} C_{j^{+}}$is the ionic strength in the outer solution. From this equation, the asymptotic expressions for $\alpha$ can be obtained for the two limiting cases:

$$
\alpha \approx \begin{cases}\left(\frac{K_{a} C_{\mathrm{i}}}{C_{\mathrm{H}} c_{\mathrm{p}}}\right)^{1 / 2} & \left(C_{\mathrm{i}} \ll \alpha c_{\mathrm{p}}\right), \\ \frac{K_{a}}{C_{\mathrm{H}}} & \left(C_{\mathrm{i}} \gg \alpha c_{\mathrm{p}}\right) .\end{cases}
$$

Here, $\alpha$ is taken to be much smaller than unity, because we consider the case in which the corona chains have weakly dissociating groups under acidic condition. In the low ionic strength limit $\left(C_{\mathrm{i}} \ll \alpha c_{\mathrm{p}}\right)$, added salt promotes the dissociation of weakly acidic groups. In the high ionic strength limit $\left(C_{\mathrm{i}} \gg \alpha c_{\mathrm{p}}\right)$, on the other hand, $\alpha$ is determined by the proton concentration in the outer solution. In the latter case, we have $c_{\mathrm{H}} \approx C_{\mathrm{H}}$ [see Eqs. (1) and (15)].

Under the electroneutrality condition, the bare electrostatic repulsion between the corona chains is screened by the counterions. The corona free energy per chain can be written as a sum of the three contributions (31):

$$
\begin{aligned}
f_{\text {corona }}= & \frac{3 L^{2}}{2 N_{\mathrm{B}} a^{2}}+\Omega_{B}\left(-\frac{1}{2} \tau a^{3} c_{\mathrm{p}}^{2}+\frac{1}{6} w a^{6} c_{\mathrm{p}}^{3}\right) \\
& +\Omega_{B}\left[\left(\sum_{i} c_{i^{-}}+\sum_{j} c_{j^{+}}\right)\left\{\log a^{3}\left(\sum_{i} c_{i^{-}}+\sum_{j} c_{j^{+}}\right)-1\right\}\right. \\
& \left.-\left(\sum_{i} C_{i^{-}}+\sum_{j} C_{j^{+}}\right)\left\{\log a^{3}\left(\sum_{i} C_{i^{-}}+\sum_{j} C_{j^{+}}\right)-1\right\}\right] .
\end{aligned}
$$


The first contribution is the elastic energy of the corona chain. The second contribution represents the nonelectrostatic interactions between the charged monomers. Notice that $\tau a^{3}$ and $w a^{6}$ denote the second and the third virial coefficients, respectively. The last contribution is the entropy loss of the counterions confined within the corona. Using Eqs. (3), (4), $(8),(9)$, and (13), $f_{\text {corona }}$ is rewritten as

$$
\begin{aligned}
\frac{f_{\text {corona }}}{N_{B}}= & \frac{3 R_{\mathrm{c}}^{2}}{2 N_{\mathrm{B}}^{2} a^{2}}\left\{\left(1+\frac{N_{B}}{a^{3} N_{A} c_{\mathrm{p}}}\right)^{1 / 3}-1\right\}^{2}-\frac{1}{2} \tau a^{3} c_{\mathrm{p}}+\frac{1}{6} w a^{6} c_{\mathrm{p}}^{2} \\
& +\left(\alpha+\frac{2 C_{\mathrm{H}}}{c_{\mathrm{H}}} \frac{C_{\mathrm{i}}}{c_{\mathrm{p}}}\right)\left\{\log a^{3}\left(\alpha c_{\mathrm{p}}+\frac{2 C_{\mathrm{H}}}{c_{\mathrm{H}}} C_{\mathrm{i}}\right)-1\right\} \\
& -\frac{2 C_{\mathrm{i}}}{c_{\mathrm{p}}}\left\{\log \left(2 C_{\mathrm{i}} a^{3}\right)-1\right\} .
\end{aligned}
$$

Collecting all the expressions for $f_{\text {core }}, f_{\text {interface }}$, and $f_{\text {corona }}$, the total free energy per chain is summarized as

$$
\begin{aligned}
\frac{f\left(\bar{R}_{\mathrm{c}}, \bar{c}_{\mathrm{p}}\right)}{N_{B}}= & \frac{3 \bar{R}_{\mathrm{c}}^{2}}{2 N_{\mathrm{A}} N_{\mathrm{B}}}\left[1+\frac{N_{A}}{N_{B}}\left\{\left(1+\frac{N_{B}}{N_{A} \bar{c}_{\mathrm{p}}}\right)^{1 / 3}-1\right\}^{2}\right] \\
& +\frac{3 \bar{\gamma}_{A}}{N_{B} \bar{R}_{\mathrm{c}}}-\frac{1}{2} \tau \bar{c}_{\mathrm{p}}+\frac{1}{6} w \bar{c}_{\mathrm{p}}^{2} \\
& +\left(\alpha+\frac{2 \bar{C}_{\mathrm{H}}}{\bar{c}_{\mathrm{H}}} \bar{C}_{\mathrm{i}}\right)\left\{\log \left(\alpha \bar{c}_{\mathrm{p}}+\frac{2 \bar{C}_{\mathrm{H}}}{\bar{c}_{\mathrm{H}}} \bar{C}_{\mathrm{i}}\right)-1\right\} \\
& -\frac{2 \bar{C}_{\mathrm{i}}}{\bar{c}_{\mathrm{p}}}\left\{\log \left(2 \bar{C}_{\mathrm{i}}\right)-1\right\} .
\end{aligned}
$$

In the above, we have used the following dimensionless quantities:

$$
\begin{gathered}
\bar{R}_{\mathrm{c}}=R_{\mathrm{c}} a^{-1}, \quad \bar{\gamma}=\gamma a^{2}, \quad \bar{c}_{i}=c_{i} a^{3}(i=\mathrm{p}, \mathrm{H}), \\
\bar{C}_{j}=C_{j} a^{3}(j=\mathrm{H}, \mathrm{i}) .
\end{gathered}
$$

Minimizing $f$ with respect to $\bar{R}_{\mathrm{c}}$, we obtain

$$
\bar{R}_{\mathrm{c}}=N_{A}^{2 / 3} \bar{\gamma}^{1 / 3}\left[1+\frac{N_{A}}{N_{B}}\left\{\left(1+\frac{N_{B}}{N_{A} \bar{c}_{\mathrm{p}}}\right)^{1 / 3}-1\right\}^{2}\right]^{-1 / 3} .
$$


Hence, the minimized free energy as a function of $\bar{c}_{\mathrm{p}}$ only is given by

$$
\begin{aligned}
\frac{f\left(\bar{c}_{\mathrm{p}}\right)}{N_{B}}= & \frac{9}{2} N_{A}^{1 / 3} N_{B}^{-1} \bar{\gamma}^{2 / 3}\left[1+\frac{N_{A}}{N_{B}}\left\{\left(1+\frac{N_{B}}{N_{A} \bar{c}_{\mathrm{p}}}\right)^{1 / 3}-1\right\}^{2}\right]^{1 / 3}-\frac{1}{2} \tau \bar{c}_{\mathrm{p}}+\frac{1}{6} w \bar{c}_{\mathrm{p}}^{2} \\
& +\left(\alpha+\frac{2 \bar{C}_{\mathrm{H}}}{\bar{c}_{\mathrm{H}}} \frac{\bar{C}_{\mathrm{i}}}{\bar{c}_{\mathrm{p}}}\right)\left\{\log \left(\alpha \bar{c}_{\mathrm{p}}+\frac{2 \bar{C}_{\mathrm{H}}}{\bar{c}_{\mathrm{H}}} \bar{C}_{\mathrm{i}}\right)-1\right\}-\frac{2 \bar{C}_{\mathrm{i}}}{\bar{c}_{\mathrm{p}}}\left\{\log \left(2 \bar{C}_{\mathrm{i}}\right)-1\right\} .
\end{aligned}
$$

In the limiting cases, the last two terms of Eq. (21) (the entropy terms) can be approximated as

$$
\frac{f_{\mathrm{e}}\left(\bar{c}_{\mathrm{p}}\right)}{N_{B}} \approx \begin{cases}-\alpha\left(1+\log \phi_{\mathrm{ci}}^{-1}\right)=-\left(1+\log \phi_{\mathrm{ci}}^{-1}\right)\left(\beta^{-1} \bar{C}_{\mathrm{i}}\right)^{1 / 2} \bar{c}_{\mathrm{p}}^{-1 / 2} & \left(\bar{C}_{\mathrm{i}} \ll \alpha c_{\mathrm{p}}\right), \\ \frac{\bar{c}_{\mathrm{p}}}{4 \beta^{2} \bar{C}_{\mathrm{i}}} & \left(\bar{C}_{\mathrm{i}} \gg \alpha c_{\mathrm{p}}\right),\end{cases}
$$

respectively, where we have used the following definitions:

$$
\phi_{\mathrm{ci}}=\alpha \bar{c}_{\mathrm{p}}, \quad \beta=C_{\mathrm{H}} / K_{a} .
$$

The approximated expressions for $f_{\mathrm{e}}$ indicate that the mobile ions in the corona induce an effective repulsion between the charged monomers. This interaction competes with the elastic energy and the nonelectrostatic attraction between the monomers.

\section{CRITICAL MICELLE CONCENTRATION}

When the copolymer concentration exceeds the critical micelle concentration $(\mathrm{CMC})$, the copolymers self-assemble to form micelles. In this section, we derive the expression for CMC by introducing the free energy of a micellar solution. In addition, we obtain the free energy of an individual copolymer in the solution.

As in the previous section, we assume that the electroneutrality condition in the corona is satisfied for each micelle. In this case, the bare electrostatic interaction between the micelles can be neglected. The total free energy of a dilute micellar solution is written as [6]

$$
F_{\text {mic }}=\sum_{p} f_{p} \phi_{p}+\sum_{p} \frac{\phi_{p}}{p}\left[\log \left(\frac{\phi_{p}}{p}\right)-1\right],
$$

where $p$ is the aggregation number as before, $\phi_{p}$ the volume fraction of copolymers packed into micelles composed of $p$ copolymers, and $f_{p}$ the micellar energy per copolymer of aggregation number $p$. When the 
formation of spherical micelles is favored, $f_{p}$ takes the minimum value for a certain aggregation number $p^{*}$. The first term of Eq. (24) represents the average energy of the micelle. The second term denotes the translational entropy of the micelles. The nonelectrostatic interactions between micelles are neglected.

To determine the optimum size distribution of charged micelles, we minimize $F_{\text {mic }}$ with respect to $\left\{\phi_{p}\right\}$ under the constraint of mass conservation:

$$
\sum_{p} \phi_{p}=\phi
$$

where $\phi$ is the total volume fraction of copolymers in the solution. Using a Lagrange multiplier method, we obtain the size distribution of micelles as

$$
\phi_{p}=p\left[\phi_{1} \exp \left(f_{1}-f_{p}\right)\right]^{p} .
$$

From this equation, the total volume fraction of copolymers is approximately written as

$$
\phi \approx \phi_{1}+\phi_{p^{*}}=\phi_{1}+p^{*}\left[\phi_{1} \exp \left(f_{1}-f_{p^{*}}\right)\right]^{p^{*}}
$$

Because $\phi_{p^{*}}<1$, we have $\phi_{1}<\exp \left(f_{p^{*}}-f_{1}\right)$. When $\phi<\exp \left(f_{p^{*}}-f_{1}\right)$, $\phi_{p^{*}}$ is negligible compared to $\phi_{1}$, whereas $\phi_{p^{*}}$ increases rapidly when $\phi>$ $\exp \left(f_{p^{*}}-f_{1}\right)$. Hence, CMC is given by

$$
\phi_{\mathrm{cmc}}=\exp \left(f_{p^{*}}-f_{1}\right) .
$$

The condition required to form micelles is $\phi_{\mathrm{cmc}}<1$.

We next derive the optimum free energy of an individual charged copolymer $f_{1}$. In the solution, the neutral $A$ block collapses, while the conformation of the charged $B$ block is a spherical coil of radius $\bar{L}_{1} a(3,4)$. Then the free energy of an unaggregated copolymer is written as

$$
f_{1}\left(\bar{L}_{1}\right)=N_{A}^{2 / 3} \bar{\gamma}+\frac{3 \bar{L}_{1}^{2}}{2 N_{B}}+ \begin{cases}-\left(N_{B} \beta^{-1} \bar{C}_{\mathrm{i}}\right)^{1 / 2} \bar{L}_{1}^{3 / 2} & \left(C_{\mathrm{i}} \ll \alpha c_{\mathrm{p}}^{\prime}\right), \\ \frac{N_{B}^{2}}{4 N_{A} \beta^{2} \bar{C}_{\mathrm{i}}} \bar{L}_{1}^{-3} & \left(C_{\mathrm{i}} \gg \alpha c_{\mathrm{p}}^{\prime}\right),\end{cases}
$$

where $c_{\mathrm{p}}{ }^{\prime}$ is the monomer concentration in the coil of the $B$ block. The first term represents the interfacial energy of the collapsed $A$ block. The second term is the elastic energy of the $B$ block. The last term is the translational entropy of the mobile ions inside the coil of the $B$ block. This term is obtained by recalling the discussion in the second section of 
this paper. Minimizing $f_{1}$ with respect to $\bar{L}_{1}$, we obtain

$$
\bar{L}_{1} \sim \begin{cases}N_{B}^{3} \beta^{-1} \bar{C}_{\mathrm{i}} & \left(\bar{C}_{\mathrm{i}} \ll N_{B}^{-2} \beta^{1 / 2}\right), \\ N_{B}^{3 / 5} \beta^{-2 / 5} \bar{C}_{\mathrm{i}}^{-1 / 5} & \left(\bar{C}_{\mathrm{i}} \gg N_{B}^{-2} \beta^{1 / 2}\right) .\end{cases}
$$

Hence, the optimum free energy of a single copolymer is

$$
F_{1}=N_{A}^{2 / 3} \bar{\gamma}+ \begin{cases}-N_{B}^{5} \beta^{-2} \bar{C}_{\mathrm{i}}^{2} & \left(\bar{C}_{\mathrm{i}} \ll N_{B}^{-2} \beta^{1 / 2}\right), \\ N_{B}^{1 / 5} \beta^{-4 / 5} \bar{C}_{\mathrm{i}}^{-2 / 5} & \left(\bar{C}_{\mathrm{i}} \gg N_{B}^{-2} \beta^{1 / 2}\right) .\end{cases}
$$

In the following arguments, the single copolymer states characterized by $\bar{C}_{\mathrm{i}} \ll N_{B}^{-2} \beta^{1 / 2}$ and $\bar{C}_{\mathrm{i}} \gg N_{B}^{-2} \beta^{1 / 2}$ are referred to as "M0" and "M1," respectively.

\section{CREW-CUT MICELLES}

In this and the next two sections, we discuss the situation in which the non-electrostatic interaction between the charged monomers can be neglected with respect to the ion-induced repulsion. Hence, we set $\tau=w=0$. In this case, the free energy of a spherical micelle is obtained from Eqs. (21) and (22) as

$$
f(u)=\frac{9}{2} N_{A}^{1 / 3} \bar{\gamma}^{2 / 3}\left(1+\frac{N_{A} u^{2}}{N_{B}}\right)^{1 / 3}+f_{\mathrm{e}}(u)
$$

where

$$
f_{\mathrm{e}}(u)= \begin{cases}-\left(1+\log \phi_{\mathrm{ci}}^{-1}\right)\left[N_{A} N_{B} \beta^{-1} \bar{C}_{\mathrm{i}}\left\{(1+u)^{3}-1\right\}\right]^{1 / 2} & \left(\bar{C}_{\mathrm{i}} \ll \alpha c_{\mathrm{p}}\right), \\ \frac{N_{B}^{2}}{4 N_{A} \beta^{2} \bar{C}_{\mathrm{i}}}\left\{(1+u)^{3}-1\right\}^{-1} & \left(\bar{C}_{\mathrm{i}} \gg \alpha c_{\mathrm{p}}\right) .\end{cases}
$$

Here we have changed the variable from $\bar{c}_{\mathrm{p}}$ to $u$, which is defined by

$$
u \equiv \frac{L}{R_{\mathrm{c}}}=\left(1+\frac{N_{B}}{N_{A} \bar{c}_{\mathrm{p}}}\right)^{1 / 3}-1
$$

Accordingly, the core radius is rewritten as

$$
\bar{R}_{c}=N_{A}^{2 / 3} \bar{\gamma}^{1 / 3}\left(1+\frac{N_{A} u^{2}}{N_{B}}\right)^{-1 / 3} .
$$

In the following, we discuss two different types of micelles, namely, the crew-cut micelles $\left(R_{\mathrm{c}} \gg L\right.$ or $\left.u \ll 1\right)$ and the star-like micelles $\left(R_{\mathrm{c}} \ll L\right.$ or $u \gg 1$ ). By minimizing $f(u)$, we find out various regimes with different 
scaling expressions for the structural parameters. These regimes are characterized by the magnitude of the ionic strength $\bar{C}_{\mathrm{i}}$ and the acid concentration $\beta$. Notice that the dissociation degree of the charged block in the outer solution is very small $\left(K_{a} / C_{\mathrm{H}}=\beta^{-1} \ll 1\right)$ because we do not add any base.

In the following, we discuss the structural parameters of the crew-cut micelles $(u \ll 1)$. We find out five different regimes $(\mathrm{C} 0, \mathrm{C} 1, \mathrm{C} 2, \mathrm{C} 3$, and $\mathrm{QN})$ characterized by the different scaling behaviors.

\section{Low Ionic Strength Limit}

Using Eqs. (32) and (33), the free energy of a crew-cut micelle in the low ionic strength limit $\left(\bar{C}_{\mathrm{i}} \ll \alpha \overline{C_{\mathrm{p}}}\right)$ is written as

$$
\begin{aligned}
f(u)= & \frac{9}{2} N_{A}^{1 / 3} \bar{\gamma}^{2 / 3}\left(1+\frac{N_{A} u^{2}}{N_{B}}\right)^{1 / 3}-\left(1+\log \phi_{\mathrm{ci}}^{-1}\right) \\
& \times\left(3 N_{A} N_{B} \beta^{-1} \bar{C}_{\mathrm{i}}\right)^{1 / 2} u^{1 / 2} .
\end{aligned}
$$

The dissociation degree increases with decreasing the acid concentration in the outer solution [see Eq. (15)], and so does the osmotic pressure exerted on the corona chain. Hence, the corona chain is extended when the acid concentration is low, and the elastic energy of the corona chain exceeds that of the core chain. Such a situation is characterized by the condition $N_{A} u^{2} / N_{B} \gg 1$. In this case (C0 regime), the free energy of a crew-cut micelle is approximated as

$$
f(u)=\frac{9}{2} N_{A}^{2 / 3} N_{B}^{-1 / 3} \bar{\gamma}^{2 / 3} u^{2 / 3}-\left(1+\log \phi_{\mathrm{ci}}^{-1}\right)\left(3 N_{A} N_{B} \beta^{-1} \bar{C}_{\mathrm{i}}\right)^{1 / 2} u^{1 / 2} .
$$

Minimization with respect to $u$ leads to

$$
u \sim N_{A}^{-1} N_{B}^{5} \bar{\gamma}^{-4} \beta^{-3} \bar{C}_{\mathrm{i}}^{3},
$$

where we have assumed that the logarithmic term is approximately constant. Using Eqs. (15), (35), and $u=\bar{L} / \bar{R}_{\mathrm{c}}$, we get

$$
\begin{aligned}
\bar{R}_{\mathrm{c}} & \sim N_{A} N_{B}^{-3} \bar{\gamma}^{3} \beta^{2} \bar{C}_{\mathrm{i}}^{-2}, \\
\bar{L} & \sim N_{B}^{2} \bar{\gamma}^{-1} \beta^{-1} \bar{C}_{\mathrm{i}}, \\
\alpha & \sim N_{B}^{2} \bar{\gamma}^{-2} \beta^{-2} \bar{C}_{\mathrm{i}}^{2} .
\end{aligned}
$$

The above results as well as the others are summarized in Table 1. From Eqs. (40) and (41), we find $\bar{L} \sim N_{B} \alpha^{1 / 2}$. This is the scaling expression for the osmotic brush with thickness determined by the balance between the elastic force and the osmotic pressure (32). The corona thickness increases with 
TABLE 1 Scaling expressions for the structural parameters for a charged spherical micelle and a unaggregated copolymer.

\begin{tabular}{llll}
\hline Regime $^{a}$ & \multicolumn{1}{c}{$\bar{R}_{\mathrm{c}}$} & \multicolumn{1}{c}{$\bar{c}\left(\bar{L}_{1}{ }^{b}\right)$} & \multicolumn{1}{c}{$\alpha$} \\
\hline C0 & $N_{A} N_{B}^{-3} \bar{\gamma}^{3} \beta^{2} \bar{C}_{\mathrm{i}}^{-2}$ & $N_{B}^{2} \bar{\gamma}^{-1} \beta^{-1} \bar{C}_{\mathrm{i}}$ & $N_{B}^{2} \bar{\gamma}^{-2} \beta^{-2} \bar{C}_{\mathrm{i}}^{2}$ \\
$\mathrm{C} 1$ & $N_{A} N_{B}^{-3 / 5} \bar{\gamma}^{3 / 5} \beta^{4 / 5} \bar{C}_{\mathrm{i}}^{2 / 5}$ & $N_{B}^{4 / 5} \bar{\gamma}^{1 / 5} \beta^{-2 / 5} \bar{C}_{\mathrm{i}}^{-1 / 5}$ & $\beta^{-1}$ \\
C2 & $N_{A}^{2 / 3} \bar{\gamma}^{1 / 3}$ & $N_{A}^{1 / 9} N_{B} \bar{\gamma}^{-1 / 9} \beta^{-1 / 3} \bar{C}_{\mathrm{i}}^{1 / 3}$ & $N_{A}^{2 / 9} \bar{\gamma}^{-2 / 9} \beta^{-2 / 3} \bar{C}_{\mathrm{i}}^{2 / 3}$ \\
C3 & $N_{A}^{2 / 3} \bar{\gamma}^{1 / 3}$ & $N_{A}^{-1} / 9 N_{B} \bar{\gamma}^{1 / 9} \beta^{-2 / 3} \bar{C}_{\mathrm{i}}^{-1 / 3}$ & $\beta^{-1}$ \\
S1 & $N_{A}^{7 / 11} N_{B}^{-1 / 11} \bar{\gamma}^{5 / 11} \beta^{4 / 11} \bar{C}_{\mathrm{i}}^{2 / 11}$ & $N_{A}^{2 / 11} N_{B}^{6 / 11} \bar{\gamma}^{3 / 11} \beta^{-2 / 11} \bar{C}_{\mathrm{i}}^{-1 / 11}$ & $\beta^{-1}$ \\
S2 & $N_{A}^{2 / 3} \bar{\gamma}^{1 / 3}$ & $N_{A}^{-1} N_{B}^{3} \bar{\gamma}^{-1} \beta^{-1} \bar{C}_{\mathrm{i}}$ & $N_{A}^{-2} N_{B}^{4} \bar{\gamma}^{-2} \beta^{-2} \bar{C}_{\mathrm{i}}^{2}$ \\
S3 & $N_{A}^{2 / 3} \bar{\gamma}^{1 / 3}$ & $N_{A}^{1 / 5} N_{B}^{3 / 5} \bar{\gamma}^{1 / 5} \beta^{-2 / 5} \bar{C}_{\mathrm{i}}^{-1 / 5}$ & $\beta^{-1}$ \\
QN & $N_{A}^{2 / 3} \bar{\gamma}^{1 / 3}$ & $N_{B}^{1 / 2}$ & $\beta^{-1} c$ \\
& & & $N_{A}^{2 / 9} \bar{\gamma}^{-2 / 9} \beta^{-2 / 3} \bar{C}_{\mathrm{i}}^{2 / 3 c}$ \\
& & & $N_{A}^{-2} N_{B}^{4} \bar{\gamma}^{-2} \beta^{-2} \bar{C}_{\mathrm{i}}^{2} c$ \\
M0 & $N_{A}^{1 / 3}$ & $N_{B}^{3} \beta^{-1} \bar{C}_{\mathrm{i}}$ & $N_{B}^{4} \beta^{-2} \bar{C}_{\mathrm{i}}^{2}$ \\
M1 & $N_{A}^{1 / 3}$ & $N_{B}^{3 / 5} \beta^{-2 / 5} \bar{C}_{\mathrm{i}}^{-1 / 5}$ & $\beta^{-1}$ \\
\hline
\end{tabular}

${ }^{a} \mathrm{C}, \mathrm{S}, \mathrm{QN}$, and M stand for crew-cut, star-like, quasi-neutral micelles and a single copolymer, respectively. ${ }^{b}$ The radius of a spherical coil composed of a charged block.

${ }^{c} \alpha$ varies according to the magnitude of $\bar{C}_{\mathrm{i}}$ and the neutral/charged relative block length (see Sec. REGIME DIAGRAMS).

the ionic strength, because the dissociation degree increases. This behavior is peculiar to the corona composed of charged blocks with weakly dissociating groups (13). For the corona with strongly dissociating groups, its thickness decreases with increasing the ionic strength due to the screening effect $(5,8,9,19,20)$. In the $\mathrm{C} 0$ regime, $u$ decreases by the addition of acid.

When $u \ll\left(N_{B} / N_{A}\right)^{1 / 2}$ or $\beta \gg N_{A}^{-1 / 6} N_{B}^{3 / 2} \bar{\gamma}^{-4 / 3} \bar{C}_{\mathrm{i}}$, the elastic energy of the core becomes dominant. In this case (C2 regime), the free energy of a crew-cut micelle is approximated as

$$
\begin{aligned}
f(u)= & \frac{9}{2} N_{A}^{1 / 3} \bar{\gamma}^{2 / 3}+\frac{3}{2} N_{A}^{4 / 3} N_{B}^{-1} \bar{\gamma}^{2 / 3} u^{2}-\left(1+\log \phi_{\mathrm{ci}}^{-1}\right) \\
& \times\left(3 N_{A} N_{B} \beta^{-1} \bar{C}_{\mathrm{i}}\right)^{1 / 2} u^{1 / 2} .
\end{aligned}
$$

Minimizing $f$ with respect to $u$, we obtain

$$
u \sim N_{A}^{-5 / 9} N_{B} \bar{\gamma}^{-4 / 9} \beta^{-1 / 3} \bar{C}_{\mathrm{i}}^{1 / 3} .
$$

Using Eqs. (15) and (35), we get

$$
\begin{aligned}
\bar{R}_{\mathrm{c}} & \sim N_{A}^{2 / 3} \bar{\gamma}^{1 / 3}, \\
\bar{L} & \sim N_{A}^{1 / 9} N_{B} \bar{\gamma}^{-1 / 9} \beta^{-1 / 3} \bar{C}_{\mathrm{i}}^{1 / 3}, \\
\alpha & \sim N_{A}^{2 / 9} \bar{\gamma}^{-2 / 9} \beta^{-2 / 3} \bar{C}_{\mathrm{i}}^{2 / 3},
\end{aligned}
$$

(see Table 1). As in the $\mathrm{C} 0$ regime, this scaling expression for the corona thickness is identical to that for the osmotic brush (32). Owing to the 
dominance of the core elastic energy, the core radius depends neither on the acid concentration nor on the ionic strength. However, the corona shrinks by the addition of acid. At $\beta \sim \beta_{2 N} \equiv N_{A}^{1 / 3} N_{B}^{3 / 2} \bar{\gamma}^{-1 / 3} \bar{C}_{\mathrm{i}}$, the corona thickness takes the minimum size $\bar{L} \sim N_{B}^{1 / 2}$, which is the radius of a neutral polymer in a $\theta$-solvent. For $\beta>\beta_{2 \mathrm{~N}}$, the structural parameters of a crew-cut micelle are constant (quasi-neutral [QN] regime).

As mentioned above, the $\mathrm{C} 0$ regime is characterized by three conditions: the low ionic strength limit $\left(\bar{C}_{\mathrm{i}} \ll \alpha \overline{\mathrm{C}_{\mathrm{p}}}\right)$, the dominance of the corona elastic energy $\left(N_{A} u^{2} / N_{B} \gg 1\right)$, and the crew-cut condition $(u \ll 1)$. Using the results of Eqs. (38) and (41), these conditions can be rewritten as

$$
\begin{aligned}
& \bar{C}_{\mathrm{i}} \ll N_{B}^{-1} \bar{\gamma} \beta^{1 / 2}, \\
& \bar{C}_{\mathrm{i}} \gg N_{A}^{1 / 6} N_{B}^{-3 / 2} \bar{\gamma}^{4 / 3} \beta, \\
& \bar{C}_{\mathrm{i}} \ll N_{A}^{1 / 3} N_{B}^{-5 / 3} \bar{\gamma}^{4 / 3} \beta .
\end{aligned}
$$

So that these conditions are simultaneously satisfied for $\beta \gg 1, N_{B}$ should be restricted to $N_{A}^{1 / 3} \bar{\gamma}^{2 / 3} \ll N_{B} \ll N_{A}$. In the case of $N_{A}^{1 / 2} \bar{\gamma}^{1 / 2} \ll N_{B} \ll N_{A}$, the transition between the crew-cut and the star-like micelles occurs when $u \sim 1$ (or, equivalently, $\bar{C}_{\mathrm{i}} \sim N_{A}^{1 / 3} N_{B}^{-5 / 3} \bar{\gamma}^{4 / 3} \beta$ ).

The four conditions characterizing the $\mathrm{C} 2$ regime are obtained by repeating the same discussion. They are summarized in Table 2. In order to fulfill these conditions simultaneously for $\beta \gg 1, N_{B}$ should be restricted to $N_{B} \ll N_{A}^{4 / 3} \bar{\gamma}^{2 / 3}$. In the case of $N_{A} \ll N_{B} \ll N_{A}^{4 / 3} \bar{\gamma}^{2 / 3}$, the transition between the crew-cut and the star-like micelles occurs when $u \sim 1$ (or, equivalently, $\bar{C}_{\mathrm{i}} \sim N_{A}^{5 / 3} N_{B}^{-3} \bar{\gamma}^{4 / 3} \beta$ ).

\section{High lonic Strength Limit}

In the limit of high ionic strength $\left(\bar{C}_{\mathrm{i}} \gg \alpha \overline{\mathrm{C}_{\mathrm{p}}}\right)$, the free energy of a crewcut micelle is written as

$$
f(u)=\frac{9}{2} N_{A}^{1 / 3} \bar{\gamma}^{2 / 3}\left(1+\frac{N_{A} u^{2}}{N_{B}}\right)^{1 / 3}+\frac{N_{B}^{2}}{12 N_{A} \beta^{2} \bar{C}_{\mathrm{i}}} u^{-1} .
$$

At low acid concentration, the elastic energy of the corona is larger than that of the core $\left(N_{A} u^{2} / N_{B} \gg 1\right)$. In this case (C1 regime), the minimization of $f(u)$ gives

$$
\begin{aligned}
\bar{R}_{c} & \sim N_{A} N_{B}^{-3 / 5} \bar{\gamma}^{3 / 5} \beta^{4 / 5} \bar{C}_{\mathrm{i}}^{2 / 5}, \\
\bar{L} & \sim N_{B}^{4 / 5} \bar{\gamma}^{1 / 5} \beta^{-2 / 5} \bar{C}_{\mathrm{i}}^{-1 / 5} .
\end{aligned}
$$

The dissociation degree is determined by the acid concentration, $\alpha=\beta^{-1}$ [see Eq. (15)]. From these results, we find $\bar{L} \sim N_{B} \cup^{1 / 3} \bar{\sigma}^{-1 / 3}$ where 
TABLE 2 Conditions for each regime.

\begin{tabular}{|c|c|c|c|}
\hline \multirow[b]{2}{*}{ Condition } & \multicolumn{3}{|c|}{ Regime } \\
\hline & C0 & C1 & $\mathrm{C} 2$ \\
\hline \multirow[t]{2}{*}{$\begin{array}{l}\text { (i) }^{a} \\
\text { (ii) }^{b} \\
\text { (iii) }^{c} \\
\text { (iv) }^{d}\end{array}$} & $\begin{aligned} & \bar{C}_{\mathrm{i}} \ll N_{A}^{1 / 3} N_{B}^{-5 / 3} \bar{\gamma}^{4 / 3} \beta \\
& \bar{C}_{\mathrm{i}} \ll N_{B}^{-1} \bar{\gamma} \beta^{1 / 2} \\
& \bar{C}_{\mathrm{i}} \gg N_{A}^{1 / 6} N_{B}^{-3 / 2} \bar{\gamma}^{4 / 3} \beta\end{aligned}$ & $\begin{array}{l}\bar{C}_{\mathrm{i}} \gg N_{A}^{-5 / 3} N_{B}^{7 / 3} \bar{\gamma}^{-2 / 3} \beta^{-2} \\
\bar{C}_{\mathrm{i}} \gg N_{B}^{-1} \bar{\gamma} \beta^{1 / 2} \\
\bar{C}_{\mathrm{i}} \ll N_{A}^{-5 / 6} N_{B}^{3 / 2} \bar{\gamma}^{-2 / 3} \beta^{-2}\end{array}$ & $\begin{aligned} \bar{C}_{\mathrm{i}} \ll N_{A}^{5 / 3} N_{B}^{-3} \bar{\gamma}^{4 / 3} \beta \\
\bar{C}_{\mathrm{i}} \ll N_{A}^{-1 / 3} \bar{\gamma}^{1 / 3} \beta^{-1 / 2} \\
\bar{C}_{\mathrm{i}} \ll N_{A}^{1 / 6} N_{B}^{-3 / 2} \bar{\gamma}^{4 / 3} \beta \\
\bar{C}_{\mathrm{i}} \gg N_{A}^{-1 / 3} N_{B}^{-3 / 2} \bar{\gamma}^{1 / 3} \beta\end{aligned}$ \\
\hline & C3 & $\mathrm{S} 1$ & $\mathrm{~S} 2$ \\
\hline \multirow[t]{2}{*}{$\begin{array}{l}\text { (i) } \\
\text { (ii) } \\
\text { (iii) } \\
\text { (iv) }\end{array}$} & $\begin{array}{l}\bar{C}_{\mathrm{i}} \gg N_{A}^{-7 / 3} N_{B}^{3} \bar{\gamma}^{-2 / 3} \beta^{-2} \\
\bar{C}_{\mathrm{i}} \ll N_{A}^{-1 / 3} \bar{\gamma}^{1 / 3} \beta^{-1 / 2} \\
\bar{C}_{\mathrm{i}} \gg N_{A}^{-5 / 6} N_{B}^{3 / 2} \bar{\gamma}^{-2 / 3} \beta^{-2} \\
\bar{C}_{\mathrm{i}} \ll N_{A}^{-1 / 3} N_{B}^{3 / 2} \bar{\gamma}^{1 / 3} \beta^{-2}\end{array}$ & $\begin{aligned} & \bar{C}_{\mathrm{i}} \ll N_{A}^{-5 / 3} N_{B}^{7 / 3} \bar{\gamma}^{-2 / 3} \beta^{-2} \\
& \bar{C}_{\mathrm{i}} \gg N_{A}^{2} N_{B}^{-5} \bar{\gamma}^{3} \beta^{7 / 2} \\
& \bar{C}_{\mathrm{i}} \ll N_{A}^{1 / 6} N_{B}^{1 / 2} \bar{\gamma}^{-2 / 3} \beta^{-2}\end{aligned}$ & $\begin{array}{l}\bar{C}_{\mathrm{i}} \gg N_{A}^{5 / 3} N_{B}^{-3} \bar{\gamma}^{4 / 3} \beta \\
\bar{C}_{\mathrm{i}} \ll N_{A} N_{B}^{-2} \bar{\gamma} \beta^{1 / 2} \\
\bar{C}_{\mathrm{i}} \ll N_{A}^{2} N_{B}^{-5} \bar{\gamma}^{3} \beta^{7 / 2} e \\
\bar{C}_{\mathrm{i}} \gg N_{A} N_{B}^{-5 / 2} \bar{\gamma} \beta\end{array}$ \\
\hline & S3 & M0 & M1 \\
\hline \multirow[t]{2}{*}{$\begin{array}{l}\text { (i) } \\
\text { (ii) } \\
\text { (iii) } \\
\text { (iv) }\end{array}$} & $\begin{aligned} & \bar{C}_{\mathrm{i}} \ll N_{A}^{-7 / 3} N_{B}^{3} \bar{\gamma}^{-2 / 3} \beta^{-2} \\
& \bar{C}_{\mathrm{i}} \gg N_{A} N_{B}^{-2} \bar{\gamma} \beta^{1 / 2} \\
& \bar{C}_{\mathrm{i}} \gg N_{A}^{1 / 6} N_{B}^{1 / 2} \bar{\gamma}^{-2 / 3} \beta^{-2} \\
& \bar{C}_{\mathrm{i}} \ll N_{A} N_{B}^{1 / 2} \bar{\gamma} \beta^{-2}\end{aligned}$ & $\mathrm{QN}\left(N_{B} \ll N_{A}^{4 / 3} \bar{\gamma}^{2 / 3}\right)$ & $\mathrm{QN}\left(N_{B} \gg N_{A}^{4 / 3} \bar{\gamma}^{2 / 3}\right)$ \\
\hline & & $\begin{array}{l}\bar{C}_{\mathrm{i}} \ll N_{A}^{-1 / 3} N_{B}^{-3 / 2} \bar{\gamma}^{1 / 3} \beta \\
\bar{C}_{\mathrm{i}} \gg N_{A}^{-1 / 3} N_{B}^{3 / 2} \bar{\gamma}^{1 / 3} \beta^{-2}\end{array}$ & $\begin{array}{l}\bar{C}_{\mathrm{i}} \ll N_{A} N_{B}^{-5 / 2} \bar{\gamma} \beta \\
\bar{C}_{\mathrm{i}} \gg N_{A} N_{B}^{1 / 2} \bar{\gamma} \beta^{-2}\end{array}$ \\
\hline
\end{tabular}

${ }^{a}$ Crew-cut micelles $(u \ll 1)$ or star-like micelles $(u \gg 1)$.

${ }^{b}$ Low ionic strength $\left(\bar{C}_{\mathrm{i}} \ll \alpha \overline{c_{\mathrm{p}}}\right)$ or high ionic strength $\left(\bar{C}_{\mathrm{i}} \gg \alpha \overline{c_{\mathrm{p}}}\right)$.

${ }^{c}$ The dominance of the elastic energy of the core $\left(N_{A} u^{2} / N_{B} \ll 1\right)$ or the corona $\left(N_{A} u^{2} / N_{B} \gg 1\right)$.

${ }^{d}$ The lower limit of the corona thickness $\left(\bar{L} \gg N_{B}^{1 / 2}\right)$.

${ }^{e}$ This relation is derived from the condition that the micellar free energy takes the minimum value as discussed in Appendix A.

$v=\alpha^{2} / \bar{C}_{\mathrm{i}}$, and $\bar{\sigma}=3 N_{A} / \bar{R}_{\mathrm{c}}$ is the surface area per grafted polymer [see Eq. (7)]. This is the scaling expression for the thickness of a planar neutral polymer brush with an effective excluded volume parameter $v=\alpha^{2} / \bar{C}_{\mathrm{i}}$ $(33,34)$. The effect of added electrolytes (salt or acid) is reflected in the excluded volume parameter, as in the case of the corona with strongly dissociating groups (8). By increasing the acid concentration, the corona shrinks because the effective excluded volume decreases. The core chains, on the other hand, are more stretched by adding acid. Eventually, the elastic energy of the core becomes dominant $\left(N_{A} u^{2} / N_{B} \ll 1\right)$. In this case (C3 regime), the structural parameters are obtained as

$$
\begin{aligned}
\bar{R}_{\mathrm{c}} & \sim N_{A}^{2 / 3} \bar{\gamma}^{1 / 3}, \\
\bar{L} & \sim N_{A}^{-1 / 9} N_{B} \bar{\gamma}^{1 / 9} \beta^{-2 / 3} \bar{C}_{\mathrm{i}}^{-1 / 3} .
\end{aligned}
$$

As in the C1 regime, the scaling expression for $\bar{L}$ is similar to that of a planar neutral polymer brush. The corona thickness decreases by the addition of acid or salt. This dependence is stronger than that in the C1 
regime, because the interfacial area per corona chain does not decrease. At $\beta \sim \beta_{3 \mathrm{~N}} \equiv N_{A}^{-1 / 6} N_{B}^{3 / 4} \bar{\gamma}^{1 / 6} \bar{C}_{\mathrm{i}}^{-1 / 2}, \bar{L}$ reaches the minimum size $N_{B}^{1 / 2}$. For $\beta>\beta_{3 \mathrm{~N}}$, neither the acid concentration nor the ionic strength affects the structural parameters ( $Q N$ regime).

The conditions for each regime are summarized in Table 2 . When all the conditions are simultaneously fulfilled for $\beta \gg 1, N_{B}$ is restricted to $N_{A}^{1 / 3} \bar{\gamma}^{2 / 3}$ $N_{B} \ll N_{A}$ for the C1 regime, and $N_{B} \ll N_{A}^{4 / 3} \bar{\gamma}^{2 / 3}$ for the C3 regime, respectively. In particular, when $N_{B}$ satisfies $N_{A}^{1 / 2} \bar{\gamma}^{1 / 2} \ll N_{B} \ll N_{A}$ in the C1 regime and $N_{A} \ll N_{B} \ll N_{A}^{4 / 3} \bar{\gamma}^{2 / 3}$ in the C3 regime, the transition between the crew-cut and the star-like micelles occurs at $u \sim 1$.

\section{STAR-LIKE MICELLES}

In this section, we discuss the structural parameters of star-like micelles $(u \gg 1)$. In the following, four different regimes $(\mathrm{S} 1, \mathrm{~S} 2, \mathrm{~S} 3$, and $\mathrm{QN})$ are described.

\section{Low lonic Strength Limit}

In the low ionic strength limit $\left(\bar{C}_{\mathrm{i}} \ll \alpha \overline{c_{\mathrm{p}}}\right)$, the free energy of a star-like micelle per chain is written from Eqs. (32) and (33) as

$$
\begin{aligned}
f(u)= & \frac{9}{2} N_{A}^{1 / 3} \bar{\gamma}^{2 / 3}\left(1+\frac{N_{A} u^{2}}{N_{B}}\right)^{1 / 3}-\left(1+\log \phi_{\mathrm{ci}}^{-1}\right) \\
& \times\left(N_{A} N_{B} \beta^{-1} \bar{C}_{\mathrm{i}}\right)^{1 / 2} u^{3 / 2} .
\end{aligned}
$$

It takes the minimum value when $\bar{C}_{\mathrm{i}} \lesssim N_{A} N_{B}^{-2} \bar{\gamma} \beta^{1 / 2}$ and $\bar{C}_{\mathrm{i}} \lesssim$ $N_{A}^{2} N_{B}^{-5} \bar{\gamma}^{3} \beta^{7 / 2}$ are simultaneously satisfied (see Appendix A). Notice that the first condition is rewritten as $\bar{C}_{\mathrm{i}} \lesssim \alpha \overline{\overline{\mathrm{p}}_{\mathrm{p}}}$. In this case (S2 regime), the structural parameters of a star-like micelle are given by using Eqs. (15), (35), and (A3) as

$$
\begin{aligned}
\bar{R}_{\mathrm{c}} & \sim N_{A}^{2 / 3} \bar{\gamma}^{1 / 3}, \\
\bar{L} & \sim N_{A}^{-1} N_{B}^{3} \bar{\gamma}^{-1} \beta^{-1} \bar{C}_{\mathrm{i}}, \\
\alpha & \sim N_{A}^{-2} N_{B}^{4} \bar{\gamma}^{-2} \beta^{-2} \bar{C}_{\mathrm{i}}^{2} .
\end{aligned}
$$

As in the C2 regime, the scaling expression for $\bar{L}$ is equal to that of the osmotic brush. When the acid concentration is increased, $\bar{L}$ decreases to its minimum size $N_{B}^{1 / 2}$. In this case, the structural parameters do not change even if the acid is further added ( $Q N$ regime).

The conditions characterizing the $\mathrm{S} 2$ regime are summarized in Table 2. When these conditions are simultaneously satisfied, $N_{B} \gg N_{A}$ 
should hold. In the range of $N_{A} \ll N_{B} \ll N_{A}^{4 / 3} \bar{\gamma}^{2 / 3}$, the transition between the $\mathrm{S} 2$ and the $\mathrm{C} 2$ regimes occurs at $u \sim 1$.

\section{High lonic Strength Limit}

In the high ionic strength limit $\left(\bar{C}_{\mathrm{i}} \gg \alpha \overline{\mathrm{c}_{\mathrm{p}}}\right)$, the micellar free energy is given by

$$
f(u)=\frac{9}{2} N_{A}^{1 / 3} \bar{\gamma}^{2 / 3}\left(1+\frac{N_{A} u^{2}}{N_{B}}\right)^{1 / 3}+\frac{N_{B}^{2}}{4 N_{A} \beta^{2} \bar{C}_{\mathrm{i}}} u^{-3} .
$$

By repeating the same discussion as in the previous section, we identify the S1, S3, and QN regimes. The structural parameters in each regime are summarized in Table 1 . The core radius increases by adding salt or acid in the $\mathrm{S} 1$ regime, while it does not change in the S3 and QN regimes. The corona thickness in the $\mathrm{S} 1$ and $\mathrm{S} 3$ regimes is given by $\bar{L} \sim N_{A}^{-1 / 5} N_{B}^{3 / 5} v^{1 / 5} \bar{R}_{\mathrm{c}}^{3 / 5}$ with $v=\alpha^{2} / \bar{C}_{\mathrm{i}}$. This scaling is similar to that of a spherical neutral polymer brush with an effective excluded volume parameter $v$. In these regimes, the corona thickness is reduced by the addition of electrolytes, which decreases the effective excluded volume. This dependence in the $\mathrm{S} 1$ regime is weaker than that in the S3 regime, because the core radius is increased in $\mathrm{S} 1$, whereas it is constant in S3.

The conditions for each regime are summarized in Table 2. The S1 and S3 regimes exist when $N_{B} \gg N_{A}^{1 / 2} \bar{\gamma}^{1 / 2}$ and $N_{B} \gg N_{A}$, respectively. In particular, if $N_{B}$ is restricted to $N_{A}^{1 / 2} \bar{\gamma}^{1 / 2} \ll N_{B} \ll N_{A}$ in $\mathrm{S} 1$ and $N_{A} \ll$ $N_{B} \ll N_{A}^{4 / 3} \bar{\gamma}^{2 / 3}$ in S3, the transition between crew-cut and star-like micelles occurs at $u \sim 1$.

\section{REGIME DIAGRAMS}

Using the results in the previous sections as summarized in Tables 1 and 2, we now construct the regime diagrams. Depending on the neutral/charged relative block length, six different types of the diagrams are obtained (see from Fig. 2 to Fig. 7). They contain various regimes with different scaling expressions for the structural parameters. As stated before, we consider the case of $\beta \gg 1$.

$$
N_{B} \ll N_{A}^{1 / 3} \bar{\gamma}^{2 / 3}
$$

Because the charged $B$ block is much shorter than the neutral $A$ block, the free energy of the $B$ chain is negligible compared to that of the $A$ chain. The core radius is determined by the competition between the elastic energy of the core and the interfacial energy. Hence, it depends neither 


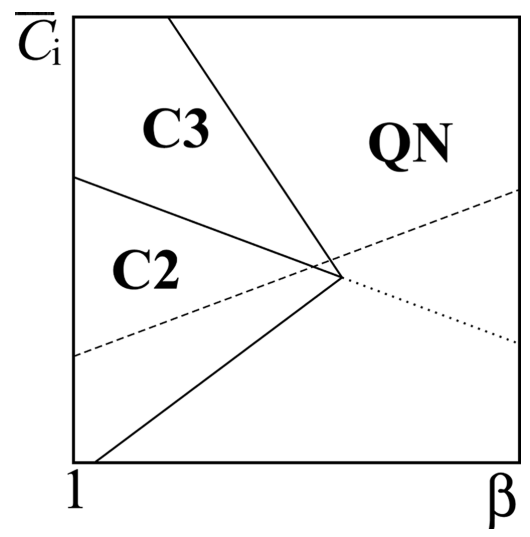

FIGURE 2 Regime diagram as a function of the ionic strength $\bar{C}_{\mathrm{i}}$ and the ratio of acid concentration to acidic dissociation constant $\beta$ for $N_{B} \ll N_{A}^{1 / 3} \bar{\gamma}^{2 / 3}$. Both axes are logarithmic. The dotted line $\left(C_{\mathrm{i}}=N_{A}^{-1 / 3} \bar{\gamma}^{1 / 3} \beta^{-1 / 2}\right)$ divides the $\mathrm{QN}$ regime into two regions. The dashed line $\left(\bar{C}_{\mathrm{i}}=\right.$ $N_{B}^{-2} \beta^{1 / 2}$ ) is the boundary between the M0 (below) and M1 (above) regions.

on the acid concentration nor on the ionic strength. In this case, only the C2, C3, and QN regimes appear as shown in Fig. 2.

When the acid concentration is relatively low, the salt concentration dependences of the corona thickness vary according to the ionic strength. At very low ionic strength, $\bar{L}$ is independent of the salt concentration and the acid concentration (QN regime). At intermediate ionic strength, $\bar{L}$ increases as $\bar{C}_{\mathrm{i}}^{1 / 3}$, because the added salt promotes the dissociation of the acidic groups (C2 regime). At high ionic strength, however, $\bar{L}$ decreases as $\bar{C}_{\mathrm{i}}^{-1 / 3}$, because the effective excluded volume is reduced (C3 regime). When the acid concentration is very high, $\bar{L}$ remains constant (QN regime). As shown by the dotted line in Fig. 2, the QN regime can be

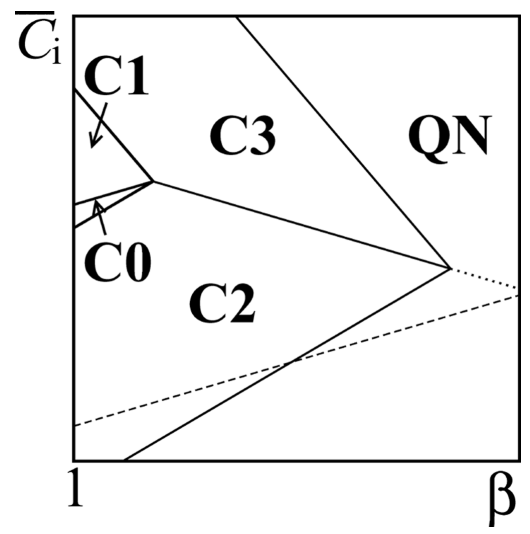

FIGURE 3 Regime diagram for $N_{A}^{1 / 3} \bar{\gamma}^{2 / 3} \ll N_{B} \ll N_{A}^{1 / 2} \bar{\gamma}^{1 / 2}$. Both axes are logarithmic. The meanings of the dotted and the dashed lines are explained in Fig. 2. 


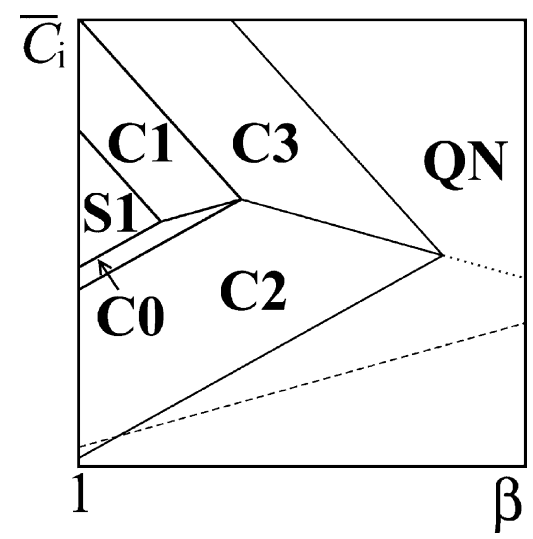

FIGURE 4 Regime diagram for $N_{A}^{1 / 2} \bar{\gamma}^{1 / 2} \ll N_{B} \ll N_{A}^{2 / 3} \bar{\gamma}$. Both axes are logarithmic. On the boundary between the $\mathrm{C} 0$ and $\mathrm{S} 1$ regimes, the structural parameters change discontinuously. The meanings of the dotted and the dashed lines are explained in Fig. 2.

divided into two regions in which $\alpha$ behaves

$$
\alpha \sim \begin{cases}N_{A}^{2 / 9} \bar{\gamma}^{-2 / 9} \beta^{-2 / 3} \bar{C}_{\mathrm{i}}^{2 / 3} & \left(\bar{C}_{\mathrm{i}} \ll N_{A}^{-1 / 3} \bar{\gamma}^{1 / 3} \beta^{-1 / 2}\right), \\ \beta^{-1} & \left(\bar{C}_{\mathrm{i}} \gg N_{A}^{-1 / 3} \bar{\gamma}^{1 / 3} \beta^{-1 / 2}\right) .\end{cases}
$$

In the C2, C3, and $\mathrm{QN}$ regimes, the micellar free energy per chain is

$$
f_{p^{*}} \sim N_{A}^{1 / 3} \bar{\gamma}^{2 / 3}
$$

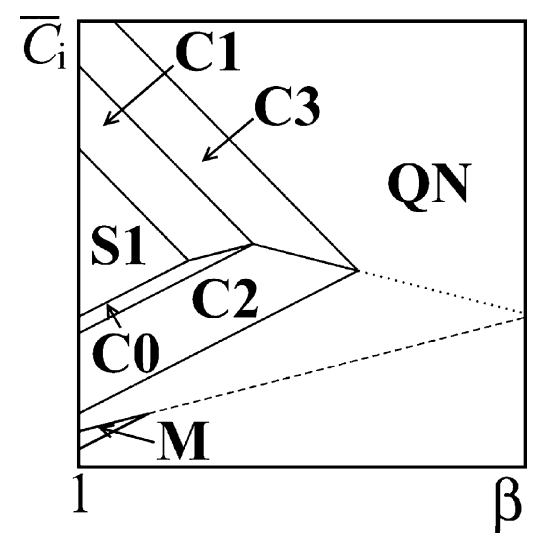

FIGURE 5 Regime diagram for $N_{A}^{2 / 3} \bar{\gamma} \ll N_{B} \ll N_{A}$. Both axes are logarithmic. In the $\mathrm{M}$ regime, charged copolymers do not aggregate into micelles. On the boundary between the C0 and S1 regimes, the structural parameters change discontinuously. The meanings of the dotted and the dashed lines are explained in Fig. 2. 


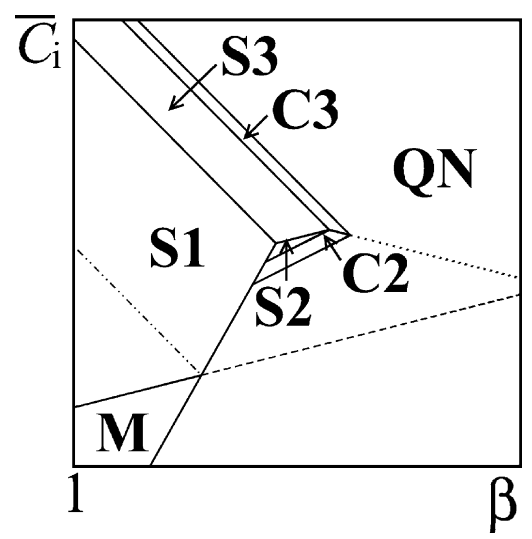

FIGURE 6 Regime diagram for $N_{A} \ll N_{B} \ll N_{A}^{4 / 3} \bar{\gamma}^{2 / 3}$. Both axes are logarithmic. The dotted and the dashed lines are explained in Fig. 2. The two-dot chain line represents $\bar{C}_{\mathrm{i}}=N_{A}^{-5 / 3} N_{B}^{1 / 2} \bar{\gamma}^{-5 / 2} \beta^{-2}$.

The free energy of an unaggregated copolymer was obtained in Eq. (31). Using Eq. (28), the CMC is given by

$$
\ln \phi_{\mathrm{cmc}} \sim \begin{cases}N_{A}^{1 / 3} \bar{\gamma}^{2 / 3}-N_{A}^{2 / 3} \bar{\gamma}+N_{B}^{5} \beta^{-2} \bar{C}_{\mathrm{i}}^{2} & \left(\bar{C}_{\mathrm{i}} \ll N_{B}^{-2} \beta^{1 / 2}\right), \\ N_{A}^{1 / 3} \bar{\gamma}^{2 / 3}-N_{A}^{2 / 3} \bar{\gamma}-N_{B}^{1 / 5} \beta^{-4 / 5} \bar{C}_{\mathrm{i}}^{-2 / 5} & \left(\bar{C}_{\mathrm{i}} \gg N_{B}^{-2} \beta^{1 / 2}\right)\end{cases}
$$

The relation $\bar{C}_{\mathrm{i}}=N_{B}^{-2} \beta^{1 / 2}$ is drawn by the dashed line in Fig. 2. The CMC in the high ionic strength limit is smaller than that in the opposite limit. The CMC decreases with increasing the acid concentration in the low ionic strength limit, while it increases in the opposite limit.

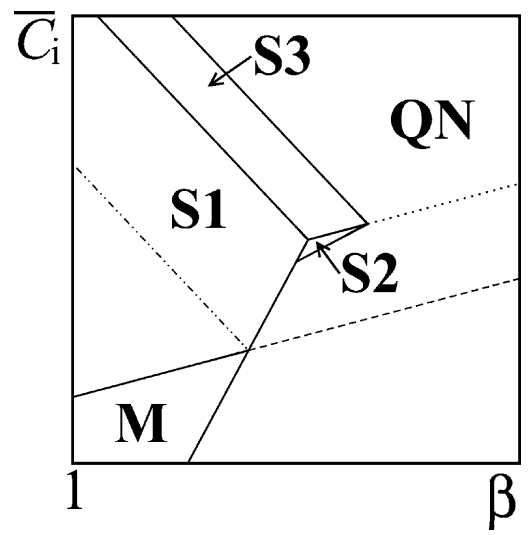

FIGURE 7 Regime diagram for $N_{B} \gg N_{A}^{4 / 3} \bar{\gamma}^{2 / 3}$. Both axes are logarithmic. The meanings of the dashed and the two-dot chain lines are explained in Fig. 2 and Fig. 6, respectively. The dotted line represents $\bar{C}_{\mathrm{i}}=N_{A} N_{B}^{-2} \bar{\gamma} \beta^{1 / 2}$. 


$$
N_{A}^{1 / 3} \bar{\gamma}^{2 / 3} \ll N_{B} \ll N_{A}^{1 / 2} \bar{\gamma}^{1 / 2}
$$

In addition to the $\mathrm{C} 2, \mathrm{C} 3$, and $\mathrm{QN}$ regimes, the $\mathrm{C} 0$ and $\mathrm{C} 1$ regimes also appear as shown in Fig. 3. The QN regime is divided into two regions characterized by the different scalings of $\alpha$ as shown in Eq. (60).

When the acid concentration is very low, the structural parameters change nonmonotonically by the addition of salt. Let us consider the sequence of $\mathrm{QN} \rightarrow \mathrm{C} 2 \rightarrow \mathrm{C} 0 \rightarrow \mathrm{C} 1 \rightarrow \mathrm{C} 3$. Then $\bar{R}_{\mathrm{c}}$ remains constant in the $\mathrm{QN}$ and $\mathrm{C} 2$ regimes and decreases as $\bar{C}_{\mathrm{i}}^{-2}$ in the $\mathrm{C} 0$ regime. In the C1 regime, it increases as $\bar{C}_{\mathrm{i}}^{2 / 5}$. In the C3 regime, it is constant, because the extension of the core chain leads to an entropic loss. On the other hand, $\bar{L}$ is constant in the QN regime. It increases as $\bar{C}_{\mathrm{i}}^{1 / 3}$ in the C2 regime and as $\bar{C}_{\mathrm{i}}$ in the $\mathrm{C} 0$ regime. In the $\mathrm{C} 1$ regime, it decreases as $\bar{C}_{\mathrm{i}}^{-1 / 5}$ and has the constant value in the C3 regime.

The addition of acid causes $\bar{R}_{\mathrm{c}}$ to increase only at low acid concentration (C0 and C1 regimes). In the other regimes, added acid does not affect $\bar{R}_{\mathrm{c}}$. When the acid concentration is increased, $\bar{L}$ decreases monotonically in the $\mathrm{C} 0, \mathrm{C} 1, \mathrm{C} 2$, and $\mathrm{C} 3$ regimes and becomes constant in the $\mathrm{QN}$ regime.

The CMC in each regime is summarized in Table 3 . Because the last term in $\ln \phi_{\mathrm{cmc}}$ is negligible in all the regimes, the lowest and the highest values of $\mathrm{CMC}$ are expected in the $\mathrm{C} 0$ and $\mathrm{C} 1$ regimes, respectively.

$$
N_{A}^{1 / 2} \bar{\gamma}^{1 / 2} \ll N_{B} \ll N_{A}^{2 / 3} \bar{\gamma}
$$

As shown in Fig. 4, the regions of small $\beta$ in the $\mathrm{C} 0$ and $\mathrm{C} 1$ regimes are replaced by the $\mathrm{S} 1$ regime. At low acid concentration, the discontinuous transition between the star-like ( $\mathrm{S} 1)$ micelles and the crew-cut $(\mathrm{C} 0)$ micelles takes place. On the transition line, the expansion ratios are given

$$
\begin{aligned}
& \frac{\bar{R}_{\mathrm{c}}^{\mathrm{C} 0}}{\bar{R}_{\mathrm{c}}^{\mathrm{S} 1}} \sim N_{A}^{-4 / 11} N_{B}^{8 / 11} \bar{\gamma}^{-4 / 11} \beta^{-6 / 11}>1, \\
& \frac{\bar{L}^{\mathrm{C} 0}}{\bar{L}^{\mathrm{S} 1}} \sim N_{A}^{2 / 11} N_{B}^{-4 / 11} \bar{\gamma}^{2 / 11} \beta^{3 / 11}<1 .
\end{aligned}
$$

TABLE 3 Critical micelle concentration for $N_{A}^{1 / 3} \bar{\gamma}^{2 / 3} \ll N_{B} \ll N_{A}^{1 / 2} \bar{\gamma}^{1 / 2}$.

\begin{tabular}{lll}
\hline Regime & \multicolumn{1}{c}{ In $\phi_{\mathrm{cmc}}+N_{A}^{2 / 3} \bar{\gamma}$} & Condition \\
\hline C0 & $-N_{B}^{3} \bar{\gamma}^{-2} \beta^{-2} \bar{C}_{\mathrm{i}}^{2}-N_{B}^{1 / 5} \beta^{-4 / 5} \bar{C}_{\mathrm{i}}^{-2 / 5}$ & \\
$\mathrm{C} 1$ & $N_{B}^{3 / 5} \bar{\gamma}^{2 / 5} \beta^{-4 / 5} \bar{C}_{\mathrm{i}}^{-2 / 5}-N_{B}^{1 / 5} \beta^{-4 / 5} \bar{C}_{\mathrm{i}}^{-2 / 5}$ & \\
C2, QN & $N_{A}^{1 / 3} \bar{\gamma}^{2 / 3}-N_{B}^{1 / 5} \beta^{-4 / 5} \bar{C}_{\mathrm{i}}^{-2 / 5}$ & $\left(\bar{C}_{\mathrm{i}} \gg N_{B}^{-2} \beta^{-1}\right)$ \\
& $N_{A}^{1 / 3} \bar{\gamma}^{2 / 3}+N_{B}^{5} \beta^{-2} \bar{C}_{\mathrm{i}}^{2}$ & $\left(\bar{C}_{\mathrm{i}} \ll N_{B}^{-2} \beta^{-1}\right)$ \\
C3 & $N_{A}^{1 / 3} \bar{\gamma}^{2 / 3}-N_{B}^{1 / 5} \beta^{-4 / 5} \bar{C}_{\mathrm{i}}^{-2 / 5}$ & \\
\hline
\end{tabular}


On the other hand, the transition between the $\mathrm{S} 1$ and $\mathrm{C} 1$ regimes is continuous.

The CMC in each regime is summarized in Table 4. These different CMCs satisfy $\phi_{\mathrm{cmc}}^{\mathrm{C} 0}<\phi_{\mathrm{cmc}}^{\mathrm{C2}} \sim \phi_{\mathrm{cmc}}^{\mathrm{C} 3} \sim \phi_{\mathrm{cmc}}^{\mathrm{QN}}<\phi_{\mathrm{cmc}}^{\mathrm{C} 1}<\phi_{\mathrm{cmc}}^{\mathrm{S} 1}$.

$$
N_{A}^{2 / 3} \bar{\gamma} \ll N_{B} \ll N_{A}
$$

In Fig. 5, there exists a regime in which the micellization does not occur (M regime). In the regime $\left(N_{A}^{1 / 3} N_{B}^{-5 / 2} \bar{\gamma}^{1 / 2} \beta \ll \bar{C}_{\mathrm{i}} \ll N_{B}^{-2} \beta^{1 / 2}\right)$, the micellar free energy $f_{p^{*}}$ and the free energy of an isolated copolymer $f_{1}$ are approximately given by

$$
\begin{aligned}
f_{p^{*}} & \sim N_{A}^{1 / 3} \bar{\gamma}^{2 / 3}, \\
f_{1} & \sim-N_{B}^{5} \beta^{-2} \bar{C}_{\mathrm{i}}^{2},
\end{aligned}
$$

respectively. Because $f_{p^{*}}$ is larger than $f_{1}$, the copolymers do not form any micelle in the M regime. Different CMCs are summarized in Table 5.

$$
N_{A} \ll N_{B} \ll N_{A}^{4 / 3} \bar{\gamma}^{2 / 3}
$$

As shown in Fig. 6, both the star-like and the crew-cut regimes exist in the diagram. In the $S 1$ regime, $\bar{R}_{\mathrm{c}}$ increases as the acid is added. Because of the entropic penalty arising from the stretched core chains, $\bar{R}_{\text {c }}$ remains constant in the S2 and S3 regimes. On the other hand, added acid can reduce $\bar{L}$ up to the size of $\bar{R}_{\mathrm{c}}$, and the transition between the star-like and the crew-cut micelles occurs. As a result, the acid concentration dependence of $\bar{L}$ becomes weaker as the $\mathrm{S} 2 \rightarrow \mathrm{C} 2$ transition takes place, while it becomes stronger as the S3 $\rightarrow$ C3 transition occurs. When the ionic strength is relatively low, the micelles transform from the $\mathrm{S} 1$ regime to the C2 or QN regimes without passing through the $\mathrm{S} 2$ regime. In this case, the structural parameters change discontinuously.

In the $\mathrm{M}$ regime $\left(N_{A}^{2} N_{B}^{-5} \bar{\gamma}^{3} \beta^{7 / 2} \ll \bar{C}_{\mathrm{i}} \ll N_{B}^{-2} \beta^{1 / 2}\right)$, the micellar free energy $f_{p^{*}}$ and the free energy of an unaggregated copolymer $f_{1}$ are

TABLE 4 Critical micelle concentration for $N_{A}^{1 / 2} \bar{\gamma}^{1 / 2} \ll N_{B} \ll N_{A}^{2 / 3} \bar{\gamma}$.

\begin{tabular}{lll}
\hline Regime & \multicolumn{1}{c}{$\ln \phi_{\mathrm{cmc}}+N_{A}^{2 / 3} \bar{\gamma}$} & Condition \\
\hline C0 & $-N_{B}^{3} \bar{\gamma}^{-2} \beta^{-2} \bar{C}_{\mathrm{i}}^{2}-N_{B}^{1 / 5} \beta^{-4 / 5} \bar{C}_{\mathrm{i}}^{-2 / 5}$ & \\
$\mathrm{C} 1$ & $N_{B}^{3 / 5} \bar{\gamma}^{2 / 5} \beta^{-4 / 5} \bar{C}_{\mathrm{i}}^{-2 / 5}-N_{B}^{1 / 5} \beta^{-4 / 5} \bar{C}_{\mathrm{i}}^{-2 / 5}$ & \\
C2,QN & $N_{A}^{1 / 3} \bar{\gamma}^{2 / 3}-N_{B}^{1 / 5} \beta^{-4 / 5} \bar{C}_{\mathrm{i}}^{-2 / 5}$ & $\left(\bar{C}_{\mathrm{i}} \gg N_{B}^{-2} \beta^{-1}\right)$ \\
& $N_{A}^{1 / 3} \bar{\gamma}^{2 / 3}+N_{B}^{5} \beta^{-2} \bar{C}_{\mathrm{i}}^{2}$ & $\left(\bar{C}_{\mathrm{i}} \ll N_{B}^{-2} \beta^{-1}\right)$ \\
$\mathrm{C} 3$ & $N_{A}^{1 / 3} \bar{\gamma}^{2 / 3}-N_{B}^{1 / 5} \beta^{-4 / 5} \bar{C}_{\mathrm{i}}^{-2 / 5}$ & \\
S1 & $N_{A}^{4 / 11} N_{B}^{1 / 11} \bar{\gamma}^{6 / 11} \beta^{-4 / 11} \bar{C}_{\mathrm{i}}^{-2 / 11}-N_{B}^{1 / 5} \beta^{-4 / 5} \bar{C}_{\mathrm{i}}^{-2 / 5}$ & \\
\hline
\end{tabular}


TABLE 5 Critical micelle concentration for $N_{A}^{2 / 3} \bar{\gamma} \ll N_{B} \ll N_{A}$.

\begin{tabular}{lll}
\hline Regime & \multicolumn{1}{c}{$\ln \phi_{\mathrm{cmc}}+N_{A}^{2 / 3} \bar{\gamma}$} & Condition \\
\hline $\mathrm{C} 0$ & $-N_{B}^{3} \bar{\gamma}^{-2} \beta^{-2} \bar{C}_{\mathrm{i}}^{2}-N_{B}^{1 / 5} \beta^{-4 / 5} \bar{C}_{\mathrm{i}}^{-2 / 5}$ & \\
$\mathrm{C} 1$ & $N_{B}^{3 / 5} \bar{\gamma}^{2 / 5} \beta^{-4 / 5} \bar{C}_{\mathrm{i}}^{-2 / 5}-N_{B}^{1 / 5} \beta^{-4 / 5} \bar{C}_{\mathrm{i}}^{-2 / 5}$ & \\
$\mathrm{C} 2, \mathrm{C} 3$ & $N_{A}^{1 / 3} \bar{\gamma}^{2 / 3}-N_{B}^{1 / 5} \beta^{-4 / 5} \bar{C}_{\mathrm{i}}^{-2 / 5}$ & \\
QN & $N_{A}^{1 / 3} \bar{\gamma}^{2 / 3}-N_{B}^{1 / 5} \beta^{-4 / 5} \bar{C}_{\mathrm{i}}^{-2 / 5}$ & $\left(\bar{C}_{\mathrm{i}} \gg N_{B}^{-2} \beta^{1 / 2}\right)$ \\
& $N_{A}^{1 / 3} \bar{\gamma}^{2 / 3}+N_{B}^{5} \beta^{-2} \bar{C}_{\mathrm{i}}^{2}$ & $\left(\bar{C}_{\mathrm{i}} \ll N_{B}^{-2} \beta^{1 / 2}\right)$ \\
S1 & $N_{A}^{4 / 11} N_{B}^{1 / 11} \bar{\gamma}^{6 / 11} \beta^{-4 / 11} \bar{C}_{\mathrm{i}}^{-2 / 11}-N_{B}^{1 / 5} \beta^{-4 / 5} \bar{C}_{\mathrm{i}}^{-2 / 5}$ & \\
\hline
\end{tabular}

approximately given by

$$
\begin{aligned}
f_{p^{*}} & \sim N_{A}^{4 / 11} N_{B}^{1 / 11} \bar{\gamma}^{6 / 11} \beta^{-4 / 11} \bar{C}_{\mathrm{i}}^{-2 / 11}, \\
f_{1} & \sim\left\{\begin{array}{lc}
N_{A}^{2 / 3} \bar{\gamma} & \left(N_{A}^{2} N_{B}^{-5} \bar{\gamma}^{3} \beta^{7 / 2} \ll \bar{C}_{\mathrm{i}} \ll N_{A}^{1 / 3} N_{B}^{-5 / 2} \bar{\gamma}^{1 / 2} \beta\right), \\
-N_{B}^{5} \beta^{2} \bar{C}_{\mathrm{i}}^{2} & \left(N_{A}^{1 / 3} N_{B}^{-5 / 2} \bar{\gamma}^{1 / 2} \beta \ll \bar{C}_{\mathrm{i}} \ll N_{B}^{-2} \beta^{1 / 2}\right) .
\end{array}\right.
\end{aligned}
$$

Because $f_{1}$ is smaller than $f_{p^{*}}$, the copolymers do not form any micelle in this regime.

We summarize the different CMCs in Table 6. In the S2, S3, C2, C3, and $\mathrm{QN}$ regimes, the CMC is approximately written as

$$
\ln \phi_{\mathrm{cmc}} \approx N_{A}^{1 / 3} \bar{\gamma}^{2 / 3}-N_{A}^{2 / 3} \bar{\gamma} \equiv \ln \phi_{\mathrm{cmc}}^{*}
$$

In the $\mathrm{S} 1$ regime, the $\mathrm{CMC}$ is roughly

$$
\ln \phi_{\mathrm{cmc}} \approx\left\{\begin{array}{cc}
-N_{B}^{1 / 5} \beta^{-4 / 5} \bar{C}_{\mathrm{i}}^{-2 / 5} \equiv \ln \phi_{\mathrm{cmc}}^{L} & \left(\bar{C}_{\mathrm{i}} \ll N_{A}^{-5 / 3} N_{B}^{1 / 2} \bar{\gamma}^{-5 / 2} \beta^{-2}\right), \\
N_{A}^{4 / 11} N_{B}^{1 / 11} \bar{\gamma}^{6 / 11} \beta^{-4 / 11} \bar{C}_{\mathrm{i}}^{-2 / 11} & \left(\bar{C}_{\mathrm{i}} \gg N_{A}^{-5 / 3} N_{B}^{1 / 2} \bar{\gamma}^{-5 / 2} \beta^{-2}\right) . \\
-N_{A}^{2 / 3} \bar{\gamma} \equiv \ln \phi_{\mathrm{cmc}}^{H} &
\end{array}\right.
$$

The condition $\bar{C}_{\mathrm{i}}=N_{A}^{-5 / 3} N_{B}^{1 / 2} \bar{\gamma}^{-5 / 2} \beta^{-2}$ is represented by the two-dot chain line in Fig. 6 . These CMCs satisfy $\phi_{\mathrm{cmc}}^{L}<\phi_{\mathrm{cmc}}^{*}<\phi_{\mathrm{cmc}}^{H}$.

TABLE 6 Critical micelle concentration for $N_{A} \gg N_{B}$.

\begin{tabular}{lll}
\hline Regime & \multicolumn{1}{c}{$\ln \phi_{\mathrm{cmc}}+N_{A}^{2 / 3} \bar{\gamma}$} & Condition \\
\hline S1 & $N_{A}^{4 / 11} N_{B}^{1 / 11} \bar{\gamma}^{6 / 11} \beta^{-4 / 11} \bar{C}_{\mathrm{i}}^{-2 / 11}-N_{B}^{1 / 5} \beta^{-4 / 5} \bar{C}_{\mathrm{i}}^{-2 / 5}$ & \\
S2, S3, C2, C3 & $N_{A}^{1 / 3} \bar{\gamma}^{2 / 3}-N_{B}^{1 / 5} \beta^{-4 / 5} \bar{C}_{\mathrm{i}}^{-2 / 5}$ & \\
QN & $N_{A}^{1 / 3} \bar{\gamma}^{2 / 3}-N_{B}^{1 / 5} \beta^{-4 / 5} \bar{C}_{\mathrm{i}}^{-2 / 5}$ & $\left(\bar{C}_{\mathrm{i}} \gg N_{B}^{-2} \beta^{1 / 2}\right)$ \\
& $N_{A}^{1 / 3} \bar{\gamma}^{2 / 3}+N_{B}^{5} \beta^{-2} \bar{C}_{\mathrm{i}}^{2}$ & $\left(\bar{C}_{\mathrm{i}} \ll N_{B}^{-2} \beta^{1 / 2}\right)$ \\
\hline
\end{tabular}




$$
N_{B} \gg N_{A}^{4 / 3} \bar{\gamma}^{2 / 3}
$$

As shown in Fig. 7, here the crew-cut micelles are not formed. In the S2 and S3 regimes, $\bar{L}$ decreases with increasing $\beta$. However, $\bar{L}$ cannot be smaller than $N_{B}^{1 / 2}$. When $\bar{L} \sim N_{B}^{1 / 2}$, the ratio between $\bar{L}$ and $\bar{R}_{\mathrm{c}}$ is

$$
\frac{\bar{L}}{\bar{R}_{\mathrm{c}}} \sim N_{A}^{-2 / 3} N_{B}^{1 / 2} \bar{\gamma}^{-1 / 3} \gg 1 .
$$

Hence, the copolymers do not aggregate into any crew-cut micelle. The QN regime is divided by the dotted line in Fig. 7. The scaling expressions of $\alpha$ in these two regions are

$$
\alpha \sim \begin{cases}N_{A}^{-2} N_{B}^{4} \bar{\gamma}^{-2} \beta^{-2} \bar{C}_{\mathrm{i}}^{2} & \left(C_{\mathrm{i}} \ll N_{A} N_{B}^{-2} \bar{\gamma} \beta^{1 / 2}\right), \\ \beta^{-1} & \left(C_{\mathrm{i}} \gg N_{A} N_{B}^{-2} \bar{\gamma} \beta^{1 / 2}\right) .\end{cases}
$$

Different CMCs are summarized in Table 6.

\section{EFFECTS OF HYDROPHOBICITY OF CHARGED BLOCKS}

In this section, we consider the situation in which the nonelectrostatic attractions between the charged monomers are important, [i.e., $\tau>0$ in Eq. (21)]. One of such attractions is the hydrophobic interaction, because the backbone of the charged block is made of hydrophobic hydrocarbons. For the micelles composed of charged copolymers possessing $\alpha$-methyl groups on the charged block, the attraction has a great effect on their structures $(35-37)$.

In Fig. 8, we plot the micellar free energy [Eq. (21)] as a function of $\overline{c_{\mathrm{p}}}$ for various values of the ionic strength $\bar{C}_{\mathrm{i}}$. For $\bar{C}_{\mathrm{i}}=1.0 \times 10^{-10}$, the free energy has only a single minimum that corresponds to the "collapsed" state of the corona chain. For $\bar{C}_{\mathrm{i}}=1.0 \times 10^{-9}$, however, the free energy has the secondary minimum corresponding to the "stretched" state. As shown in Fig. 9, the stretched state is determined by the balance between the stretching energy and the translational entropy of the counterions, while the collapsed state is dominated by the nonelectrostatic interaction. As the ionic strength is further increased, the minimum at the stretched state decreases as shown in Fig. 8(b). When the two minimum values are equal (middle curve), a first-order transition from the collapsed state to the stretched state occurs. At the transition point, the corona thickness and the dissociation degree increase discontinuously, while the core radius shrinks abruptly. In the following, we discuss how the structural parameters depend on the electrolyte concentration by minimizing the micellar free energy numerically. 

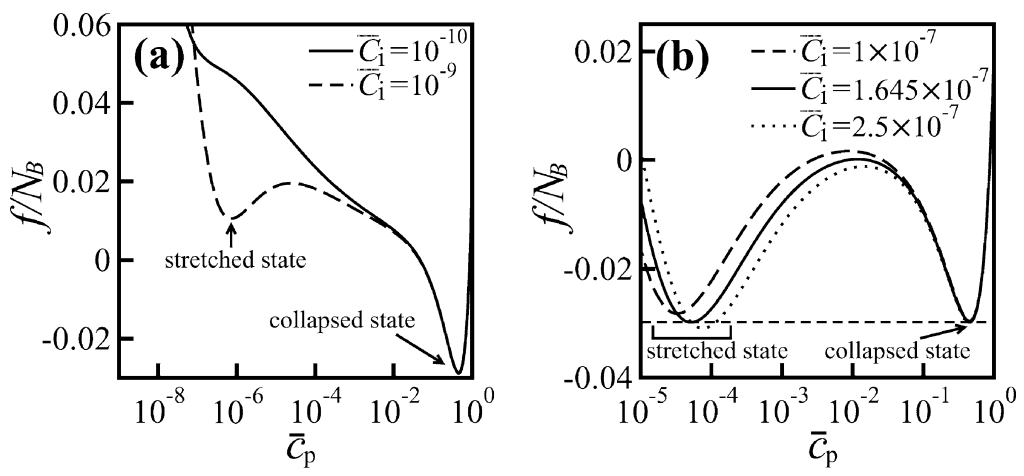

FIGURE 8 Free energy of a micelle for various values of the ionic strength. The parameters are $N_{A}=10^{3}, N_{B}=10^{4}, \bar{\gamma}=1, \tau=0.3, w=1$, and $\beta=40$. The abscissa axis is logarithmic.

\section{Effects of Added Salt}

First, we investigate the effects of added salt on the structural parameters for fixed values of the acid concentration $\beta$. We minimize the micellar free energy numerically with respect to $\bar{c}_{\mathrm{p}}$ or $\alpha$. In Fig. 10, we show the dependences of the structural parameters on the ionic strength for various values of $\beta$. As the ionic strength is increased, the structural parameters return to the original values after two discontinuous changes. This reentrant behavior arises due to the competition between the nonelectrostatic attraction among the charged monomers and the osmotic pressure induced by the mobile ions. At low ionic strength, the structural parameters do not depend on the ionic strength, because the binary attraction overwhelms the osmotic pressure. The dissociation degree increases with the ionic strength in this regime as shown in Fig. 11, and the osmotic pressure

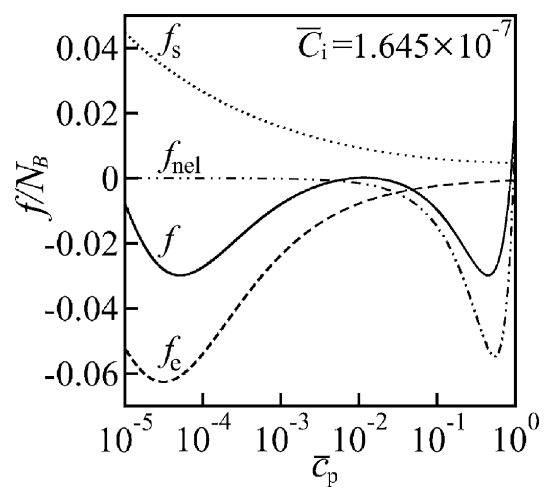

FIGURE 9 Contributions to the micellar free energy at the first-order transition point $\left(\bar{C}_{\mathrm{i}}=1.645 \times 10^{-7}\right) \cdot f_{\mathrm{s}}$ denotes the stretching energy of the copolymer, $f_{\text {nel }}$ the nonelectrostatic interaction between the charged monomers, $f_{\mathrm{e}}$ the translational entropy of the mobile ions, and $f$ the total micellar free energy. The other parameters are the same as in Fig. 8. The abscissa axis is logarithmic. 


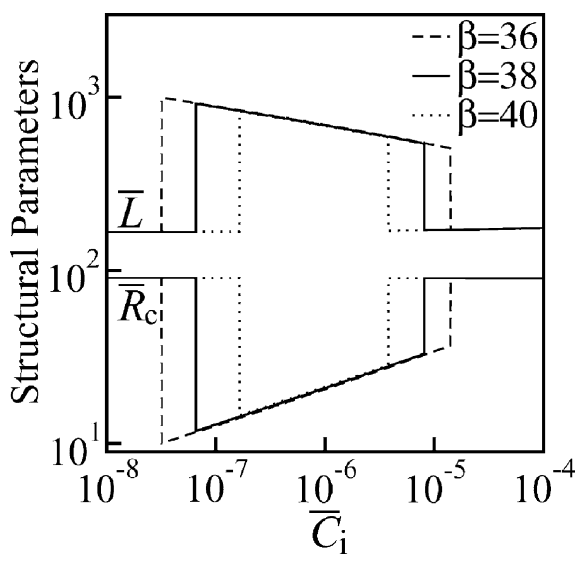

FIGURE 10 Reentrant changes of the structural parameters induced by added salt for three values of $\beta$. The parameters are $N_{A}=10^{3}, N_{B}=10^{4}, \bar{\gamma}=1, \tau=0.3$, and $w=1$. Both axes are logarithmic.

becomes larger. When these two competing interactions balance each other, the first-order transition from the collapsed micelles to the stretched ones takes place.

In the stretched state, the corona thickness decreases with increasing $\bar{C}_{\mathrm{i}}$. This behavior is attributed to the screening effect on the charged corona as detailed in Appendix B. Because the repulsion between the charged monomers decreases due to the screening effect, the core radius increases so that the core-corona interfacial energy per chain is reduced. Eventually, the osmotic repulsion balances again with the nonelectrostatic attraction, and the micelles select the collapsed state.

Fig. 10 also indicates that the transition point is dependent on the value of $\beta$. The range of $\bar{C}_{\mathrm{i}}$ in the stretched state is narrower as $\beta$ increases.

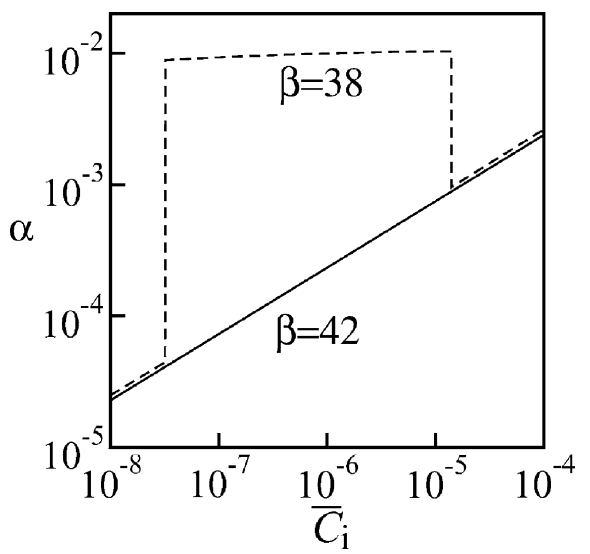

FIGURE 11 The dependences of the dissociation degree on the ionic strength for two values of $\beta$. The other parameters are the same as in Fig. 10. Both axes are logarithmic. 
Therefore, for high acid concentration, the structural parameters remain unchanged. Notice that, for $\beta=42$ in Fig. 11, the dissociation degree increases monotonically without any discontinuous jump.

\section{Effects of Added Acid}

We next discuss the effects of added acid on the micellar structure for fixed values of the salt concentration defined by $\bar{C}_{\mathrm{S}}=\bar{C}_{\mathrm{i}}-\bar{K}_{a} \beta$. Here we consider these effects not only under acidic condition but also under basic condition. In Figs. 12 and 13, the structural parameters and the dissociation degree are plotted as a function of the acid concentration for different values of the salt concentration. At very low acid concentration (achieved by the addition of base), the structural parameters are almost constant. Here, the weakly acidic groups on the corona chains are completely dissociated. When the dissociation degree decreases at around $\beta \sim 10^{-3}$ in Fig. 13, the structural parameters start to change nonmonotonically. By the addition of acid, the corona thickness increases up to the maximum value and then shrinks as shown in Fig. 12. When the osmotic repulsion balances with the nonelectrostatic attraction, the abrupt transformation from the stretched corona to the collapsed corona occurs. At the transition point, the higher the salt concentration is, the smaller the changes of the structural parameters are. For high salt concentration, the structural parameters change continuously (see the case of $\bar{C}_{\mathrm{S}}=10^{-3}$ in Fig. 12). When $\beta$ is very large, the structural parameters are only weakly dependent on the acid concentration.

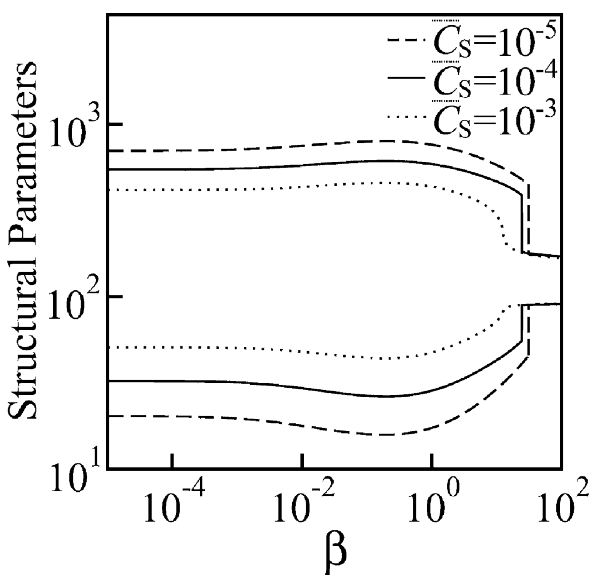

FIGURE 12 Effects of added acid on the structural parameters for three values of the salt concentration. The parameters are $N_{A}=10^{3}, N_{B}=10^{4}, \bar{\gamma}=1, \tau=0.3, w=1$, and $\bar{K}_{a}=10^{-6}$. Both axes are logarithmic. 


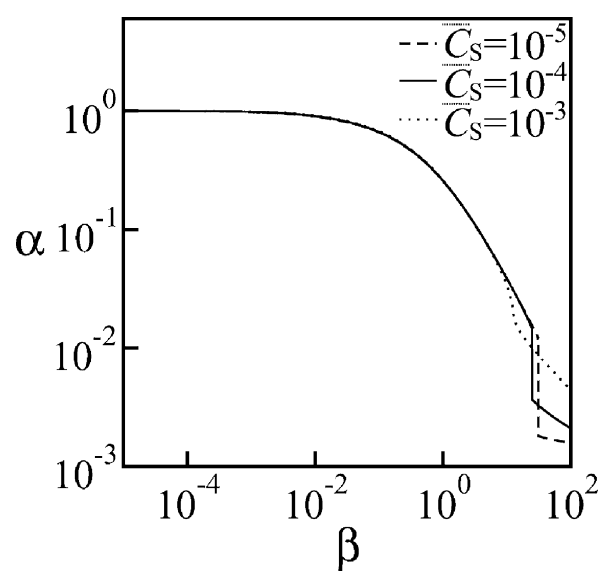

FIGURE 13 Effects of added acid on the dissociation degree for three values of the salt concentration. The parameters are the same as in Fig. 12. Both axes are logarithmic.

\section{CONCLUSIONS AND DISCUSSION}

In this paper, we investigated the effects of added electrolytes on spherical charged micelles with weakly acidic groups on the corona chains. First, we discussed the case in which the nonelectrostatic interaction between charged monomers can be neglected compared to the ion-induced repulsion (the $\theta$-solvent case). Depending on the neutral/ charged relative block length, we obtained six different types of the regime diagrams. These diagrams contain various regimes characterized by the different scaling expressions for the structural parameters. Highly asymmetric copolymers with a very short charged block form crew-cut micelles (see Figs. 2 and 3). The effects of added salt on the structural parameters at low acid concentration depend on the ionic strength; the core radius decreases with increasing the salt concentration at relatively low ionic strength, while it increases at high ionic strength. At very high and low ionic strength, however, the core radius is independent of the salt concentration. On the other hand, highly asymmetric copolymers with a very long charged block form star-like micelles (see Fig. 7). In the low acid concentration regime, added salt causes the core radius to increase and the corona thickness to decrease. These dependences are weaker than those of the crew-cut micelles. Unlike the case of the crew-cut micelles, there is no regime where the core radius decreases with increasing the salt concentration. When both the acid concentration and the ionic strength are very low, the copolymers do not aggregate. As long as the difference in the polymerization between the two blocks is not too large, the charged copolymers aggregate to form star-like micelles at relatively low acid concentration, or crew-cut micelles at high acid concentration (see Figs. 4 to 6). In the low acid concentration regime, 
the star-like micelles change discontinuously to the crew-cut micelles by the addition of acid.

Second, we investigated the charged micelles composed of copolymers with a very hydrophobic charged block (the poor solvent case). In this case, the micellar free energy has two local minima corresponding to the "stretched" and "collapsed" states of the corona chains. We predict a reentrant change of the structural parameters as the salt concentration is increased at constant acid concentration. When only acid is added, on the other hand, the nonmonotonic change of the structural parameters is expected. The corona thickness increases up to the maximum value and then decreases continuously, followed by a discontinuous stretched-to-collapsed transition. These changes arise due to the competition between the hydrophobic attraction and the ion-induced repulsion.

Previously, the structure of charged micelles with weakly acidic groups on the corona chains were investigated theoretically by Zhulina et al. $(10,11)$. They discussed the formation of spherical micelles in a solvent that is poor for the neutral blocks and marginally good for the charged blocks. For asymmetric copolymers with a very long charged block, they found that star-like charged micelles $\left(\mathrm{S}_{\mathrm{ch}}\right)$ and star-like quasi-neutral micelles $\left(\mathrm{S}_{\mathrm{qn}}\right)$ are formed depending on the ionic strength and the acid concentration. At intermediate ionic strength and low acid concentration, the formation of the $S_{c h}$ micelles was predicted. Note that the $S_{\mathrm{ch}}$ micelles are equivalent to the $\mathrm{S} 1$ micelles in the present paper. At high acid concentration, the copolymers aggregate into the $S_{\mathrm{qn}}$ micelles of large aggregation number. Notice that the $S_{\mathrm{qn}}$ micelles correspond to the QN micelles in our paper, but the scaling expressions for the structural parameters are different. For example, the core radius of the $S_{\mathrm{qn}}$ micelles depends not only on the neutral block length but also on the charged block length. Zhulina et al. also found that the addition of salt can induce a reentrant change $\mathrm{S}_{\mathrm{qn}} \rightarrow \mathrm{S}_{\mathrm{ch}} \rightarrow \mathrm{S}_{\mathrm{qn}}$. Unlike our result, the transformation $\mathrm{S}_{\mathrm{ch}} \rightarrow \mathrm{S}_{\mathrm{qn}}$ is continuous, because the nonelectrostatic interactions between the charged monomers were taken to be repulsive in Ref. (10). Another difference is that there is no regime where the elastic energy of the core affects the structural parameters as predicted for the S2 and S3 micelles. This is because the nonelectrostatic repulsion prevents the corona thickness from being too small. For asymmetric copolymers with very long neutral blocks, however, there are regimes where the elastic energy of the core affects the structural parameters [CC2 micelles in Ref. (10)]. This is in agreement with our result.

The effects of added acid on the core radius of crew-cut micelles are in qualitative agreement with the experiment on the micelles composed of highly asymmetric poly(styrene- $b$-acrylic acid) (PS-PAA) copolymers with a long PS block $(22,23)$. As the acid concentration was increased, the 
core size increased rapidly. In addition, the experiment found that the effect of added acid was stronger than that of added salt. This observation is in agreement with our analysis (see the scaling of $\bar{R}_{\mathrm{c}}$ in the $\mathrm{C} 1$ regime). At high acid concentration, however, there is a disagreement. Although we predicted the core radius to be constant at high acid concentration (C2 and C3 micelles), the experiment found that the morphology of the micelles changed after the increase in the core size.

For star-like spherical micelles of poly(styrene- $b$-sodium acrylate) (PSPAANa) copolymers, the effects of added salt have been investigated using the static light scattering measurement (18). They found that the aggregation number (or the core radius) increased as a function of the salt concentration, but it remained constant above ca. $0.10 \mathrm{M} \mathrm{NaCl}$. This behavior can be explained within our scaling analysis. Because the added salt reduces the electrostatic repulsion among the charged chains, the core radius becomes larger so that the core-corona interfacial energy per chain is reduced. For high salt concentration, however, the core does not grow due to the entropic loss of the core chains. The reentrant transition has not yet been observed in the experiment. The reason may be that the hydrophobic attraction between PAANa monomers is much smaller than the electrostatic repulsion.

Recently, the aggregation behavior of poly(methyl methacrylate- $b$ methacrylic acid) (PMMA- $b$-PMAA) copolymers over the course of neutralization was investigated (35). At low neutralization degree of PMAA chains, the copolymers with a long PMAA block formed spherical micelles and large compound micelles (LCMs). When the neutralization degree was increased, LCMs was destroyed to form spherical micelles with a more extended corona. We have reproduced this behavior qualitatively within our numerical calculation. The corona transforms from a collapsed state to an extended state as a result of the competition between the hydrophobic attraction and the ion-induced repulsion. The experiment also showed that the micellar size was nearly constant at high neutralization degree, which is in agreement with our result.

\section{APPENDIX A: S2 REGIME}

In this appendix, we derive the condition that the free energy of a starlike micelle $f(u)$ [Eq. (55)] takes the minimum value in the low ionic strength limit. Depending on the magnitude of $\bar{C}_{i}, f(u)$ either decreases monotonically or takes the local minimum and maximum values. The first derivative of $f(u)$ is

$$
\frac{\mathrm{d} f(u)}{\mathrm{d} u} \approx A_{0} u^{1 / 2}\left\{\left(\frac{A_{1}}{u^{5 / 4}+A_{2} u^{-3 / 4}}\right)^{2 / 3}-1\right\},
$$


where

$$
\begin{aligned}
& A_{0}=\frac{3}{2}\left(1+\log \phi_{\mathrm{ci}}^{-1}\right)\left(N_{A} N_{B} \beta^{-1} \bar{C}_{\mathrm{i}}\right)^{1 / 2}, \\
& A_{1}=\left(\frac{1+\log \phi_{\mathrm{ci}}^{-1}}{2}\right)^{-3 / 2} N_{A}^{1 / 4} N_{B}^{-5 / 4} \bar{\gamma} \beta^{3 / 4} \bar{C}_{\mathrm{i}}^{-3 / 4}, \\
& A_{2}=\frac{N_{B}}{N_{A}}
\end{aligned}
$$

Here, we have assumed that the logarithmic term is approximately constant.

When $A_{1} A_{2}^{-5 / 8} B<1$ with $B=(5 / 8)(3 / 5)^{3 / 8}, \mathrm{~d} f(u) / \mathrm{d} u<0$ for any $u>0$. When $A_{1} A_{2}^{-5 / 8} B>1$ or

$$
\bar{C}_{\mathrm{i}}<(5 / 4)(27 / 5)^{1 / 6}\left(1+\log \phi_{\mathrm{ci}}^{-1}\right)^{-2} N_{A}^{7 / 6} N_{B}^{-5 / 2} \bar{\gamma}^{4 / 3} \beta,
$$

$f(u)$ takes the local minimum and maximum values. From $\mathrm{d} f(u) / \mathrm{d} u=0$, we have

$$
\begin{aligned}
& u_{\operatorname{lmin}} \sim N_{A}^{-5 / 3} N_{B}^{3} \bar{\gamma}^{-4 / 3} \beta^{-1} \bar{C}_{\mathrm{i}}, \\
& u_{\operatorname{lmax}} \sim N_{A}^{1 / 5} N_{B}^{-1} \bar{\gamma}^{4 / 5} \beta^{3 / 5} \bar{C}_{\mathrm{i}}^{-3 / 5}
\end{aligned}
$$

Here, we have used the following condition given by calculating $\mathrm{d}^{2} f(u) / \mathrm{d} u^{2}$ :

$$
u_{\operatorname{lmin}}<\left(3 A_{2} / 5\right)^{1 / 2}<u_{\operatorname{lmax}}
$$

Note that $u$ is restricted to

$$
u<u_{0}=N_{A}^{-1 / 3} N_{B}^{1 / 3} \beta^{-1 / 3} \bar{C}_{\mathrm{i}}^{-1 / 3} .
$$

The inequality is obtained from Eq. (12). When $u_{\operatorname{lmin}}<u_{0}<u_{\operatorname{lmax}}$ or

$$
\bar{C}_{\mathrm{i}} \lesssim N_{A} N_{B}^{-2} \bar{\gamma} \beta^{1 / 2} \quad \text { and } \quad \bar{C}_{\mathrm{i}} \lesssim N_{A}^{2} N_{B}^{-5} \bar{\gamma}^{3} \beta^{7 / 2},
$$

$f(u)$ takes the minimum value at $u=u_{\text {lmin. }}$ Equation (A2) holds when these conditions are simultaneously satisfied. The global minimum does not exist when $u_{0}>u_{\text {lmax }}$, because $f\left(u_{\text {lmin }}\right)>f\left(u_{0}\right)$. To be exact, this relation is satisfied for $\beta \ll N_{A}^{-1 / 3} N_{B} \bar{\gamma}^{-2.3}$. However, this condition is not taken into account carefully, because our aim is to obtain the asymptotic expression for the structural parameters. Thus, $f(u)$ takes the minimum value when the condition Eq. (A7) holds. 


\section{APPENDIX B: SCREENING EFFECT}

For $\tau>0$, the addition of salt decreases the corona thickness at intermediate ionic strength. This behavior is attributed to the screening effect on the corona. In the stretched state, the micellar free energy is written as

$$
\frac{f\left(\bar{c}_{\mathrm{p}}\right)}{N_{B}} \approx \frac{9}{2} N_{A}^{4 / 9} N_{B}^{-10 / 9} \bar{\gamma}^{2 / 3} \bar{c}_{\mathrm{p}}^{-2 / 9}+\frac{1}{2}\left(\frac{1}{2 \beta^{2} \bar{C}_{\mathrm{i}}}-\tau\right) \bar{c}_{\mathrm{p}},
$$

where we have used $\bar{c}_{\mathrm{p}} \ll 1, \bar{R}_{c} \ll \bar{L}$ and $\alpha \bar{c}_{\mathrm{p}} \ll \bar{C}_{\text {i. }}$. Minimizing $f$ with respect to $\bar{c}_{\mathrm{p}}$ and using Eqs. (4) and (20), we obtain the scaling expression $\bar{L} \sim N_{A}^{-1 / 5} N_{B}^{3 / 5} \bar{R}_{\mathrm{c}}^{3 / 5} v_{\mathrm{eff}}^{1 / 5}$ with $v_{\mathrm{eff}}=\left(2 \beta^{2} \bar{C}_{\mathrm{i}}\right)^{-1}-\tau$. This expression indicates that the corona shrinks, because the added salt decreases the effective volume $v_{\text {eff }}$, which is the screening effect on the charged corona.

\section{ACKNOWLEDGMENTS}

This research is partly supported by the Grant-in-Aid for Scientific Research (C), Japan Society for the Promotion of Science under Grant No. 15540395.

\section{REFERENCES}

[1] Hamley, I.W. (1998) The Physics of Block Copolymers; Oxford University Press: Oxford.

[2] Föorster, S., Abetz, V., and Möuller, A.H.E. (2004) Adv. Polym. Sci., 166: 173.

[3] Marko, J.F. and Rabin, Y. (1992) Macromolecules, 25: 1503.

[4] Wittmer, J. and Joanny, J.F. (1993) Macromolecules, 26: 2691.

[5] Dan, N. and Tirrell, M. (1993) Macromolecules, 26: 4310.

[6] Shusharina, N.P., Nyrkova, I.A., Khokhlov, A.R. (1996) Macromolecules, 29: 3167; Shusharina, N.P., Saphonov, M.V., Nyrkova, I.A., Khalatur, P.G., and Khokhlov, A.R. (1996) Ber. Bunsenges. Phys. Chem., 100: 857.

[7] Shusharina, N.P., Linse, P., and Khokhlov, A.R. (2000) Macromolecules, 33: 3892.

[8] Netz, R.R. (1999) Europhys. Lett., 47: 391.

[9] Borisov, O.V. and Zhulina, E.B. (2002) Macromolecules, 35: 4472.

[10] Zhulina, E.B. and Borisov, O.V. (2002) Macromolecules, 35: 9191.

[11] Zhulina, E.B. and Borisov, O.V. (2005) Macromolecules, 38: 6726.

[12] Rühe, J., Ballauff, M., Biesalski, M., Dziezok, P., Gröhn, F., Johannsmann, D., Houbenov, N., Hugenberg, N., Konradi, R., Minko, S., Motornov, M., Netz, R.R., Schmidt, M., Seidel, C., Stamm, M., Stephan, T., Usov, D., and Zhang, H. (2004) Adv. Polym. Sci., 165: 79.

[13] Zhulina, E.B., Birshtein, T.M., and Borisov, O.V. (1995) Macromolecules, 28: 1491.

[14] Lyatskaya, Yu.V., Leermakers, F.A.M., Fleer, G.J., Zhulina, E.B., and Birshtein, T.M. (1995) Macromolecules, 28: 3562.

[15] Guo, X. and Ballauff, M. (2001) Phys. Rev. E, 64: 051406.

[16] Zhang, H. and Rühe, J. (2005) Macromolecules, 38: 4855.

[17] Baines, F.L., Billingham, N.C., Armes, S.P. (1996) Macromolecules, 29: 3416; Baines, F.L., Armes, S.P., Billingham, N.C., and Tuzar, Z. (1996) Macromolecules, 29: 8151.

[18] Khougaz, K., Astafieva, I., and Eisenberg, A. (1995) Macromolecules, 28: 7135.

[19] Guenoun, P., Davis, H.T., Tirrell, M., and Mays, J.W. (1996) Macromolecules, 29: 3965.

[20] Förster, S., Hermsdorf, N., Böttcher, C., and Lindner, P. (2002) Macromolecules, 35: 4096.

[21] Matsuoka, H., Maeda, S., Kaewsaiha, P., and Matsumoto, K. (2004) Langmuir, 20: 7412. 
[22] Zhang, L., Yu, K., Eisenberg, A. (1996) Science, 272: 1777; Zhang, L., and Eisenberg, A. (1996) Macromolecules, 29: 8805 .

[23] Shen, H., Zhang, L., and Eisenberg, A. (1999) J. Am. Chem. Soc., 121: 2728.

[24] Choucair, A. and Eisenberg, A. (2003) Eur. Phys. J. E, 10: 37.

[25] Förster, S., Hermsdorf, N., Leube, W., Schnablegger, H., Regenbrecht, M., Akari, S., Lindner, P., Böttcher, C. (1999) J. Phys. Chem. B, 103: 6657; Regenbrecht, M., Akari, S., Förster, S., and Möhwald, H. (1999) J. Phys. Chem. B, 103: 6669.

[26] Matsumoto, K., Ishizuka, T., Harada, T., and Matsuoka, H. (2004) Langmuir, 20: 7270.

[27] Halperin, A. (1987) Macromolecules, 20: 2943.

[28] Birshtein, T.M. and Zhulina, E.B. (1989) Polymer, 30: 170.

[29] Donnan, F.G. (1924) Chem. Rev., 1: 73.

[30] Overbeek, J.Th.G. (1956) Prog. Biophys. and Biophys. Chem., 6: 57.

[31] Ross, R.S. and Pincus, P. (1992) Macromolecules, 25: 2177.

[32] Pincus, P. (1991) Macromolecules, 24: 2912.

[33] Alexander, S. (1977) J. Phys. (Paris), 38: 983.

[34] de Gennes, P.G. (1980) Macromolecules, 13: 1069.

[35] Yao, J., Ravi, P., Tam, K.C., and Gan, L.H. (2004) Langmuir, 20: 2157.

[36] Pinteala, M., Epure, V., Harabagiu, V., Simionescu, B.C., and Schlick, S. (2004) Macromolecules, 37: 4623 .

[37] Matějíček, P., Podhájecká, K., Humpolíčková, J., Uhlík, F., Jelínek, K., Limpouchová, Z., Procházka, K., and Špírková, M. (2004) Macromolecules, 37: 10141. 